\title{
Impact response of additively manufactured metallic hybrid lattice materials
}

\author{
J.A. Harris ${ }^{\mathrm{a}}$, R.E. Winter ${ }^{\mathrm{b}}$, G.J. McShane ${ }^{*, \mathrm{a}}$ \\ ${ }^{a}$ Department of Engineering, University of Cambridge, Trumpington Street, Cambridge CB2 1PZ, UK \\ ${ }^{b} A W E$, Aldermaston, Reading, Berkshire, $U K$
}

\begin{abstract}
Additive manufacturing (AM) enables the design of new cellular materials for blast and impact mitigation by allowing novel material-geometry combinations to be realised and examined at a laboratory scale. However, design of these materials requires an understanding of the relationship between the AM process and material properties at different length scales: from the microstructure to geometric feature rendition to overall dynamic performance. To date, there remain significant uncertainties about both the potential benefits and pitfalls of using AM to design and optimise cellular materials for dynamic energy absorbing applications. This experimental investigation focuses on the out-of-plane compression of stainless steel cellular materials fabricated using selective laser melting (SLM), and makes two specific contributions. First, we demonstrate how the AM process itself influences the characteristics of these cellular materials across a range of length scales, and, crucially, how this influences the dynamic deformation. Secondly, we demonstrate how an AM route can be used to add geometric complexity to the cell structure, creating a versatile basis for future geometry optimisation. Starting with an AM square honeycomb (the reference case), we add porosity to the walls by replacing them with a lattice truss, while maintaining the same relative density. This geometry hybridisation is an approach uniquely suited to this manufacturing route. It is found that the hybrid lattice-walled honeycomb geometry significantly outperforms previously reported AM lattices in terms of specific strength, specific energy absorption, and energy absorption efficiency. It is also found that the hybrid geometry outperforms the benchmark metallic square honeycomb in terms of energy absorption efficiency in the intermediate impact velocity regime (i.e. between quasi-static loading and loading rates at which wave propagation effects begin to become pronounced), a regime in which the collapse is dominated by dynamic buckling effects.
\end{abstract}

Key words: Additive manufacturing, selective laser melting, cellular structures, blast, impact

\section{Introduction}

Mitigating the effects of dynamic loads, such as impact or blast, is an important design consideration in a range of industries and applications; defence, sporting and personal protective equipment, packaging, and automotive structures are but a few of particular interest. An ideal protective material or structure for this loading scenario is one that dissipates the kinetic energy imparted by the blast or impact event at minimum weight, while preventing the maximum stresses on the object to be protected from exceeding some critical damage or injury threshold [1, 2]. This design

* Corresponding author

Email addresses: jah236@cam.ac.uk (J.A. Harris), g jm31@cam.ac.uk (G.J. McShane) 
trade-off between energy absorption and strength is encapsulated in the energy absorption diagram method of [3].

Cellular materials have been extensively studied for blast protection and impact energy absorption applications [1, 4-9]. The large densification strains, high specific strengths, and potential for smooth and consistent plateau stresses through the deformation make them an attractive solution. Cellular materials offer a range of design variables for optimising energy absorption, including the topology (foam, honeycomb or lattice material, for example), cell size and the cell wall material. These choices affect not only the stiffness and compressive strength, but also the post-buckling characteristics up to densification. This optimisation also requires an understanding of the dynamic compressive response. The dynamic compressive strength of cellular materials is influenced by both material strain rate sensitivity and inertia effects [1, 10,-12]. The relative importance of these effects was described by Calladine and English [10]. Structures which exhibit softening during compression as a result of buckling (denoted type II in [10]) are more sensitive to inertial effects than those that collapse at near constant (or even increasing) force (denoted type I). A number of experimental and numerical investigations have been performed to examine the dynamic collapse of cellular materials fabricated from a range of materials [7, 11, 13,-16], and their findings are consistent with the predictions of [10].

The focus of the present work will be on additively manufactured metallic cellular materials. Additive manufacturing processes are finding an increasing range of applications, from miniaturised experimental components to full-scale end-use aerospace structures, due to increasing process capabilities and decreasing costs. For cellular materials, additive manufacturing processes offer a number of advantages for optimisation compared to traditional fabrication routes. Investment casting methods enable complex lattice geometries to be produced (refer to [17] for a description of this route). However, compatible alloys often have inferior mechanical properties to their wrought equivalents. The process is also unsuitable for low relative density lattices due to the flow restrictions imposed by the thin channels required [17, 18]. Sheet forming methods have been developed for the fabrication of lattice materials using wrought alloys [17, 18] but these require metallic bonding at joints, and can impose large plastic strains on the material during forming, both of which may affect ductility. Additive manufacturing (AM) processes offer the prospect of greater geometric freedom than casting processes, with alloys typical of wrought processes.

A number of AM processes are suitable for fabricating metallic cellular materials. One approach is to use polymer AM to create a scaffold onto which nanocrystalline metal is plated [19, 20]; subsequently removing the polymer core produces a low relative density hollow lattice [19, 21, 22]. Selective laser melting (SLM) allows direct additive manufacturing of metals including stainless steel, titanium alloys, and nickel-based superalloys - all of which are utilised in aerospace and other high performance applications. Low relative density lattices have been manufactured with this process in both stainless steel [7, 23-27] and titanium alloy [28, 29]. To date, studies of the impact performance of additively manufactured cellular structures have been focused primarily on lattice materials with a body-centred-cubic form (or derivatives thereof), both for blast protection [7, 24] and drop-weight impact resistance [26]. Lattice materi- 
als of various types produced by electron beam melting were tested dynamically in [30] and progressive collapse and shock effects were observed with high speed imagery, supported by Hopkinson bar stress-time data. These investigations have demonstrated the ability of AM to manufacture protective structures with geometries that were previously impractical or impossible to build. The greater uniformity and repeatability of the collapse response, for example compared to metallic foams, is noted by these authors as an additional advantage of the AM lattices. These AM lattice structures, however, did not show significant advantages in terms of specific energy absorption. McKown et al. [24] measure up to $3.7 \mathrm{~kJ} / \mathrm{kg}$ (to $50 \%$ compressive strain), and Smith et al. [7] $6.4 \mathrm{~kJ} / \mathrm{kg}$ for BCC-Z lattices, values which would be comparable to an open-celled foam of the same cell wall material and relative density [1, 2]. Stainless steel square honeycombs, on the other hand, can achieve around $25 \mathrm{~kJ} / \mathrm{kg}$ or more [31]. How to exploit AM processing capabilities in order to optimise cellular materials for impact protection, and to deliver performance in excess of traditionally manufactured equivalents, is an issue that remains to be resolved.

The aims of this study are two-fold. First, we aim to understand how the characteristics that the additive manufacturing process imparts to cellular materials influence their impact response. These characteristics include, at the micro-structural scale, grain structure and grain-level defects, influenced by the alloy and AM processing conditions. At the meso-scale, the as-manufactured geometry of the cellular material, and any geometric defects are similarly influenced by the AM process. At the macro-scale, these characteristics will interact to influence the strength, postbuckling collapse response and strain rate dependence of the structure. We also aim to investigate the extent to which miniaturisation - attractive for laboratory studies of the material response - exacerbates issues of geometry resolution, and the consequences for mechanical performance.

Second, we aim to investigate how the freedom offered by AM to realise more complex cell geometries can be used to manipulate the dynamic energy absorbing characteristics of a cellular material. This understanding can provide a basis for future efforts to exploit AM to optimise the cellular architecture for blast and impact protection. To this end, we compare a traditional cellular material - the square honeycomb - with a non-traditional, more geometricallycomplex, AM-specific alternative: a square honeycomb with the solid walls replaced by a lattice truss, while retaining the same relative density. This hybrid solution increases the number of geometric degrees of freedom, which lends itself to future use as a versatile basis for performance optimisation. It also has scope to alter the buckling characteristics of the structure, which may in turn alter the loading rate sensitivity and energy absorbing performance. Here we aim to assess whether the impact characteristics of the hybrid lattice-walled honeycomb are attractive compared to the traditional counterpart, and whether they warrant detailed optimisation.

\section{Specimen geometry}

\subsection{The hybrid lattice concept}

We take as our starting point - and reference case - the square honeycomb cellular geometry: Figure 1 1 a). This is a convenient benchmark material, as it has been investigated previously for its energy absorbing qualities, and has 
been shown to have a high specific stiffness and strength [31, 32]. This cellular structure is anisotropic, but these mechanical properties are greatest when loaded out-of-plane (ie. parallel to the prismatic direction). This is due to alignment of material with the loading direction and the constraint against buckling provided by adjacent walls. We therefore focus on out-of-plane loading of the square honeycomb as the benchmark for this investigation. Dynamic deformation out-of-plane will also be the most relevant to practical applications in structural protection, such as energy absorbing cladding or sandwich panel cores.

Honeycombs loaded in this manner can, however, undergo softening on compression, due the onset of cell wall buckling and joint tearing [31]. This softening reduces the ratio of energy absorption to peak strength, which is one measure of efficiency for impact mitigation applications. Lattice materials with a small cell size exhibit less severe softening on compression, due to progressive buckling, albeit at the expense of a lower specific energy absorption [7]. One strategy to address this is to insert additional bars into the lattice aligned with the loading direction, for example the BCC-Z lattice tested by [7].

Additive manufacturing readily enables the combination of these two classes of cellular geometries. In this study, we consider a hybrid concept: replacing the solid walls of the square honeycomb with a planar lattice truss (similar to the BCC-Z in geometry), as shown in Figure 1 (b). The bars of the lattice truss are of circular cross section with diameters chosen to keep the relative density the same as the square honeycomb. Like the square honeycomb, this lattice structure will be anisotropic. However, to allow fair comparison, we again consider loading in the out-of-plane direction, i.e. parallel to the lattice walls.

\subsection{Chosen test specimen configurations}

The three test specimen configurations considered in this investigation are shown in Figure 1(a)-(c). The test specimens fit within a circular cylindrical envelope of diameter $D=20 \mathrm{~mm}$. This dimension was chosen to maximise the contact area with the Hopkinson bar used for dynamic testing, which will be detailed later. The reference square honeycomb, Figure 1 (a), was chosen to have a cell size $L=2 \mathrm{~mm}$, so as to give 10 cells across the diameter, minimising edge effects. A simple lattice trimming method was used to fit the arrangement of square cells into a conformal cylinder [33]. The lattice-walled honeycomb hybrid specimen, abbreviated to LW-SHC in the following, was then obtained by replacing the solid walls with an array of bars, as shown in Figure 1) (b). The height of the specimen $H$ was chosen to be $10 \mathrm{~mm}$, to give five lattice unit cells in the direction of loading. This fixes the aspect ratio of the honeycomb cells to be $L / H=0.2$. It also provides a specimen aspect ratio appropriate for dynamic testing [34]. One of the objectives of this study is to assess the capabilities and limitations of the AM processing route, a key aspect of which is the minimum resolvable feature size. In order to assess the scope for miniaturisation, and the consequences for impact performance, a second lattice-walled honeycomb is specified with the unit cell size and lattice truss bar diameter reduced by a factor of two (i.e. $L=1 \mathrm{~mm}$ ), while maintaining the same values of $D, H$ and relative density. This is denoted the fine lattice-walled honeycomb (FLW-SHC) and is shown in Figure 1. (c). 


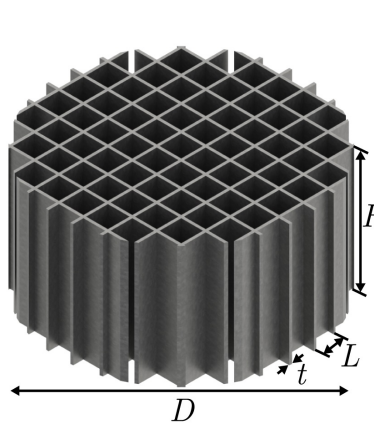

(a) Square Honeycomb (SHC)

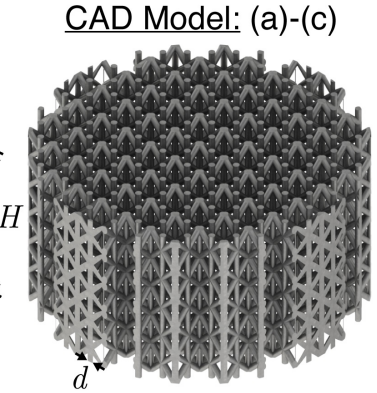

(b) Lattice-Walled Square Honeycomb (LW-SHC)

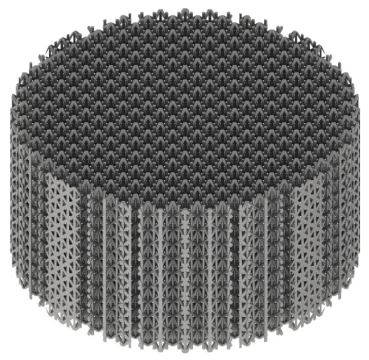

(c) Fine Lattice-Walled Square Honeycomb (FLW-SHC)

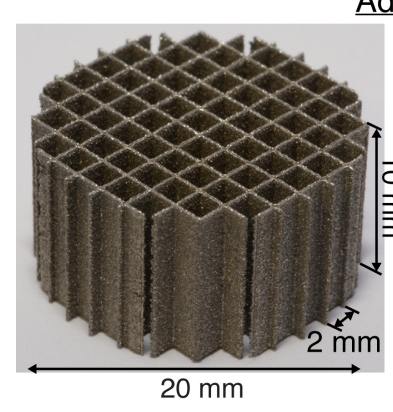

(e) Square Honeycomb (SHC)

Additively Manufactured: (e)-

- $(g)$

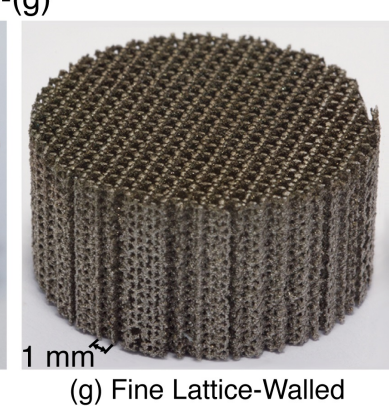

(f) Lattice-Walled Square Honeycomb (LW-SHC)
Square Honeycomb (FLW-SHC)

Figure 1: (a)-(c) CAD models of the nominal specimen geometries, and (e)-(g) photographs of the specimens as manufactured. Selected dimensions are labelled. The full set of dimensions are listed in Table 1

The final parameter to fix is the relative density of the specimens, set by the wall thickness in the square honeycomb case $(t)$, and by the truss bar diameters $(d)$ in the lattice-walled honeycomb cases. The minimum value of these parameters is set by the ability of the AM process to resolve the minimum overall dimension: the bar diameter $d$ for the FLW-SHC specimen. We opted for a value $d=0.20 \mathrm{~mm}$ for this dimension, which is approximately twice the nominal laser spot size used in the selective laser melting process (SLM, as detailed below). This results in a nominal relative density of $\bar{\rho}_{\text {nom. }} \approx 0.211$ In order to match this relative density, we specify the bar diameter $d=0.40 \mathrm{~mm}$ for the LW-SHC specimen, and the wall thickness $t=0.22 \mathrm{~mm}$ for the square honeycomb (SHC) specimen. The precise values of nominal relative density determined from the CAD models, and the experimental relative densities determined from the measured specimen masses, are quoted in Table 1.

\section{Additively manufactured specimens}

An EOS M 280 selective laser melting system was used with stainless steel 316L powder (of particle size 20-40 $\mu \mathrm{m})$ and a nominal laser spot size of $100 \mu \mathrm{m}$. The build direction was aligned with the central axis of the cylindrical specimens. All specimens were tested as manufactured, with no subsequent heat treatment or surface finishing. Ex-

\footnotetext{
${ }^{1}$ Here, we define the nominal relative density $\bar{\rho}_{\text {nom. }}$ to be that obtained from a CAD representation of the specimen. The relative density asmanufactured, obtained by weighing the test specimens, is denoted $\bar{\rho}$. The nominal and measured relative densities may differ, as described subsequently. The specimen density $\rho=\bar{\rho} \rho_{s}$, where $\rho_{s}=8000 \mathrm{~kg} \mathrm{~m}^{-3}$ is the density of conventionally manufactured solid 316L stainless steel [35]. This value of $\rho_{s}$ has been shown to be within $1 \%$ of the SLM processed equivalent using appropriate laser parameters [36].
} 


\begin{tabular}{l|ccc} 
& SHC & LW-SHC & FLW-SHC \\
\hline$D[\mathrm{~mm}]$ & 20.00 & 20.00 & 20.00 \\
$H[\mathrm{~mm}]$ & 10.00 & 10.00 & 10.00 \\
$L[\mathrm{~mm}]$ & 2.00 & 2.00 & 1.00 \\
$t$ or $d[\mathrm{~mm}]$ & 0.22 & 0.40 & 0.20 \\
$\bar{\rho}_{\text {nom. }}$ & 0.210 & 0.205 & 0.207 \\
\hline $\bar{\rho}$ & 0.212 & 0.195 & 0.256
\end{tabular}

Table 1: Nominal dimensions, nominal relative densities $\bar{\rho}_{\text {nom. }}$ and measured as-manufactured relative densities $\bar{\rho}$ (as defined in the text) of the SLM specimens. Note that $t$ refers to the wall thickness in the SHC case, and $d$ to the strut diameter in the two lattice cases.

amples of manufactured specimens of each type are shown in Figures 11(d)-(f). We proceed to discuss the key features of the AM specimens, with respect to three length scales of interest, as defined in Figure 2.
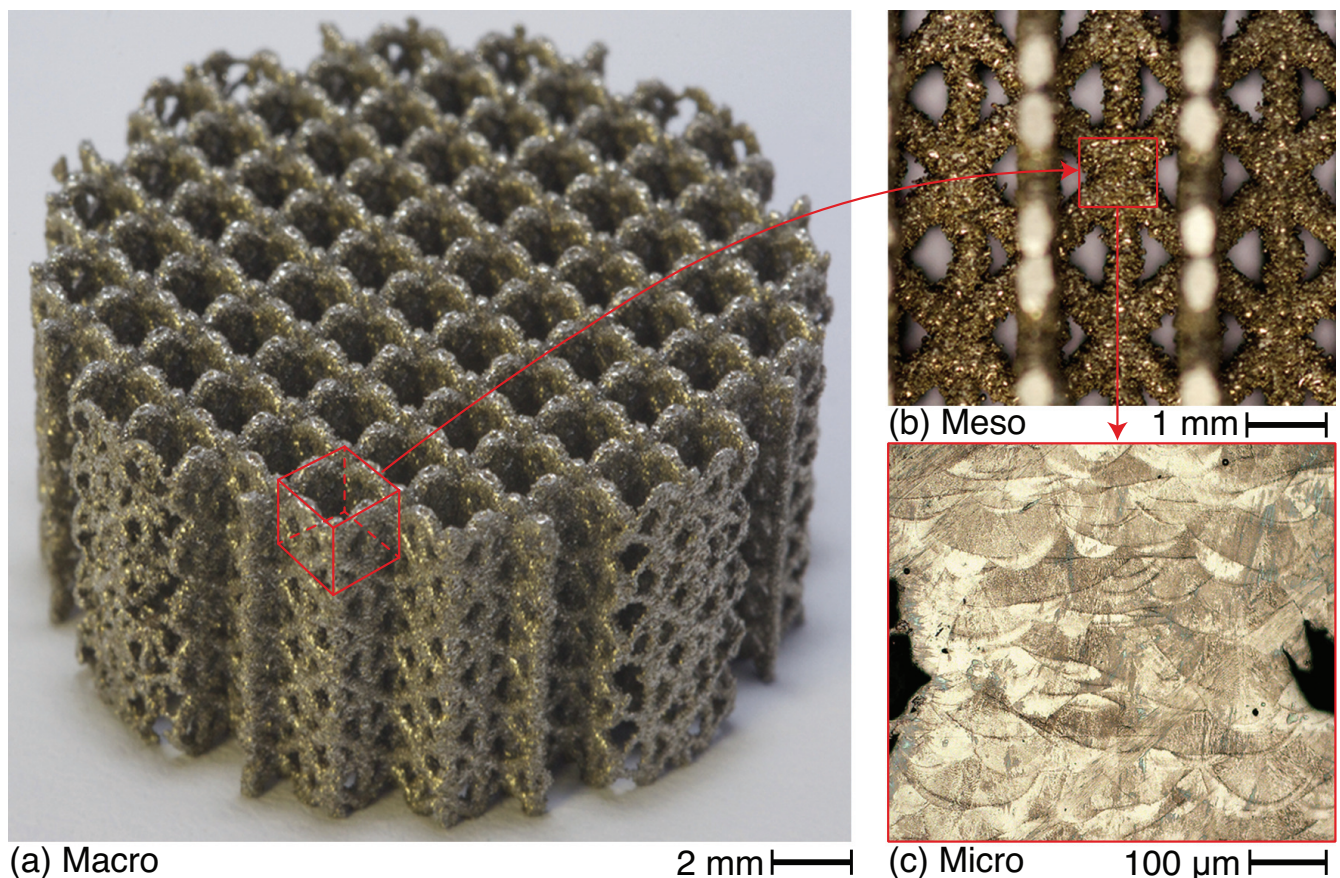

Figure 2: Length scales of interest in the manufactured specimens. (a) The macro-scale encompasses the complete cellular test specimen. b) The meso-scale refers to the details of individual bars and cell walls. c) The micro-structure includes the grain- and melt-pool level features. The melt pool boundaries are the curved features of the order $100 \mu \mathrm{m}$ in size. The grain and sub-grain structures within these melt pool features are shown in Figure 3 below.

\subsection{Micro-scale}

The microstructure of the cell wall material will influence its mechanical properties, and hence the macroscopic response of the cellular structure. It has been noted previously that laser-melted metals differ in microstructure to their wrought equivalents due to the thermal gradients imposed by the laser passes [37-40]. It will be shown subsequently that the hardness of the laser melted $316 \mathrm{~L}$ stainless steel used in this investigation is higher than that of the wrought equivalent, indicating microstructural differences. To investigate this, a LW-SHC specimen was sectioned through the wall thickness, polished, and etched with Glyceregia for one minute to reveal the microstructure: Figures 3 (a)-(c). This was repeated for a tensile dogbone specimen (as used to obtain mechanical property data) manufactured using the same SLM process, to check for consistency: Figures 3 (d)-(e). 
Dark field optical microscopy and scanning electron microscopy (SEM) images are shown in Figures 3 a)-(c), at different levels of magnification. The curved features in Figure 3 (a) represent the melt pool boundaries, and are approximately $100 \mu \mathrm{m}$ in size, comparable with the laser spot size. Multiple grains are apparent within each melt pool, as shown in Figure 3(b). Grain boundaries are observed to span the melt pool boundary, indicating epitaxial growth. While the melt pool features appear to be elongated perpendicular to the build direction (the direction of laser heating), the grains within them appear to be more equiaxed. This is the case for both the LW-SHC and dogbone specimens. Further magnification shows columnar subgrain structures - Figure 3(c). These subgrain features have previously been attributed to the rapid cooling associated with laser-based processes such as SLM [37.40]. These fine subgrain features might be expected to increase the hardness of the steel, as discussed in more detail in Section 4.1. The grain sizes range from approximately 5-15 $\mu \mathrm{m}$, with the subgrain structures being of the order 0.1-2 $\mu \mathrm{m}$.

Note that these microstructural features are the natural outcome of the SLM process, with process parameters geared towards the production of geometrically accurate parts. No attempt was made to manipulate the microstructure via the processing parameters, the potential for which is discussed by [41]. Also, as mentioned previously, no subsequent heat treatment or other post-processing was carried out in this investigation.
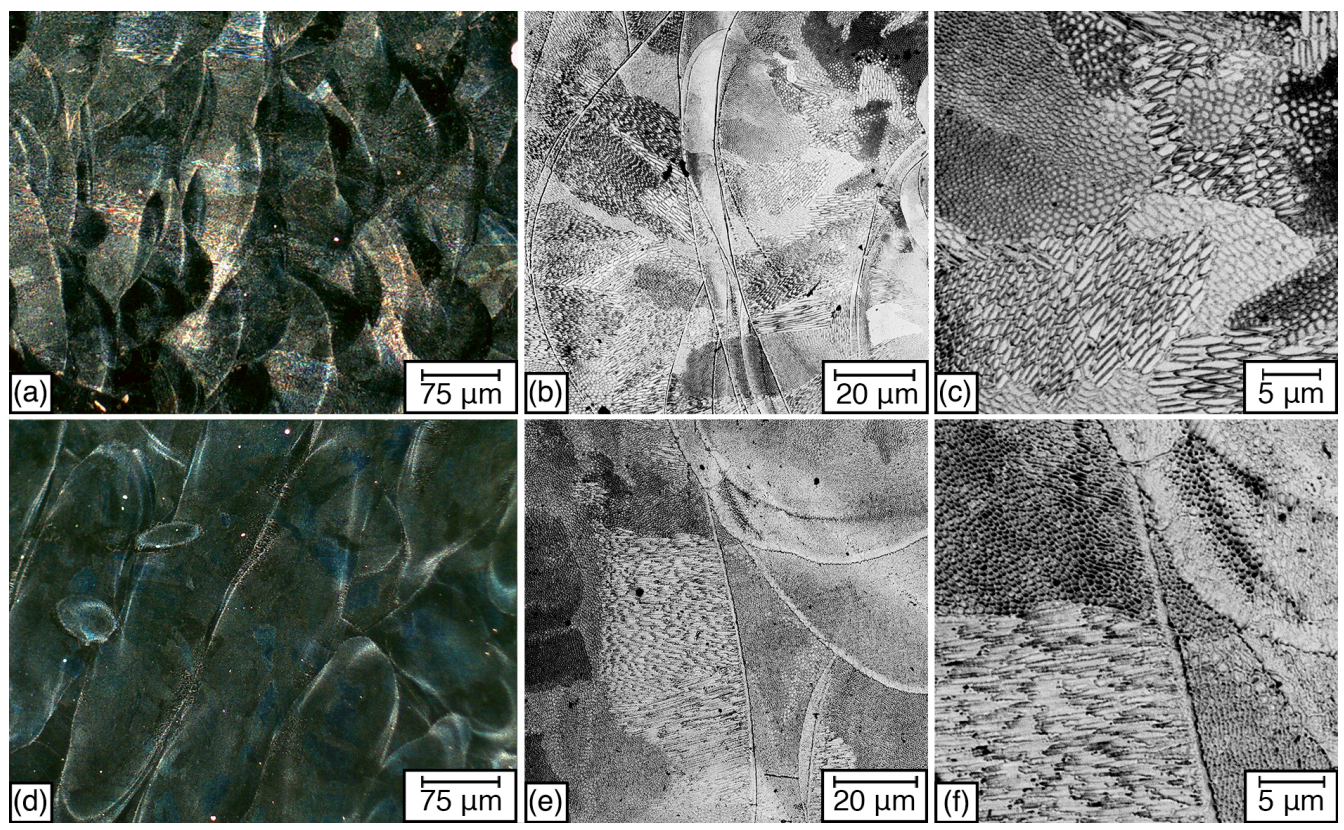

Figure 3: Optical dark field (a,d) and SEM (b,c,e,f) micrographs of: (a-c) an etched LW-SHC specimen, and (d-f) a dogbone tensile coupon, at varying levels of magnification. Similar microstructures are apparent for the two specimen types, including the presence of subgrain solidification structures.

\subsection{Meso-scale}

The as-manufactured lattice wall structure of the LW-SHC is shown in Figure 4 a). Two defects are apparent. First, a number of the vertical bars show some curvature. An example is shown magnified in Figure 4(b). The consistent direction of curvature of all defective struts suggests that it may be a consequence of some aspect of the manufacturing process. A distribution of deformed struts may reduce the compressive collapse strength of the lattice structure. 


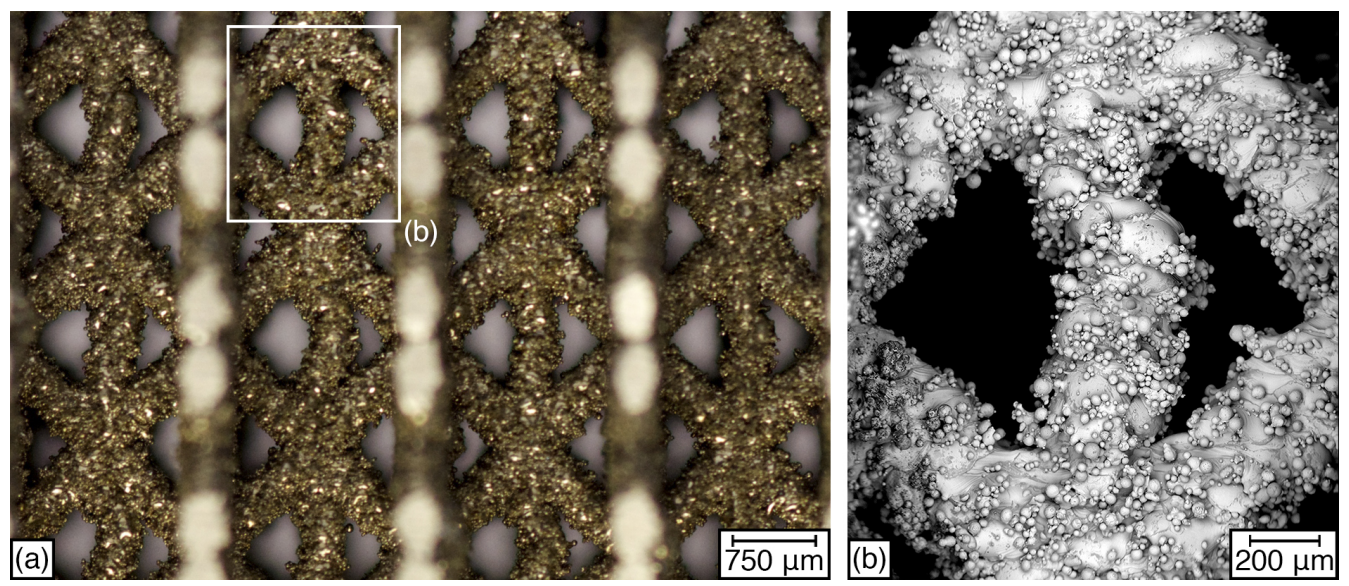

Figure 4: (a) Macro photograph of an untested, sectioned LW-SHC specimen showing numerous pre-curved vertical struts. (b) Scanning electron micrograph of an untested LW-SHC showing a curved strut and excess powder deposits.

The second defect is also shown in Figure 4 (b): the adhesion of excess powder particles to the surface of the struts. The nominal strut geometry of the LW-SHC is shown as an overlay in Figure 5 (a). This indicates that for these strut dimensions, the excess material does not significantly exceed the nominal bounds (a strut diameter of $400 \mu \mathrm{m}$ ). It appears that the parasitic mass contribution of these particles is therefore not substantial in this case.
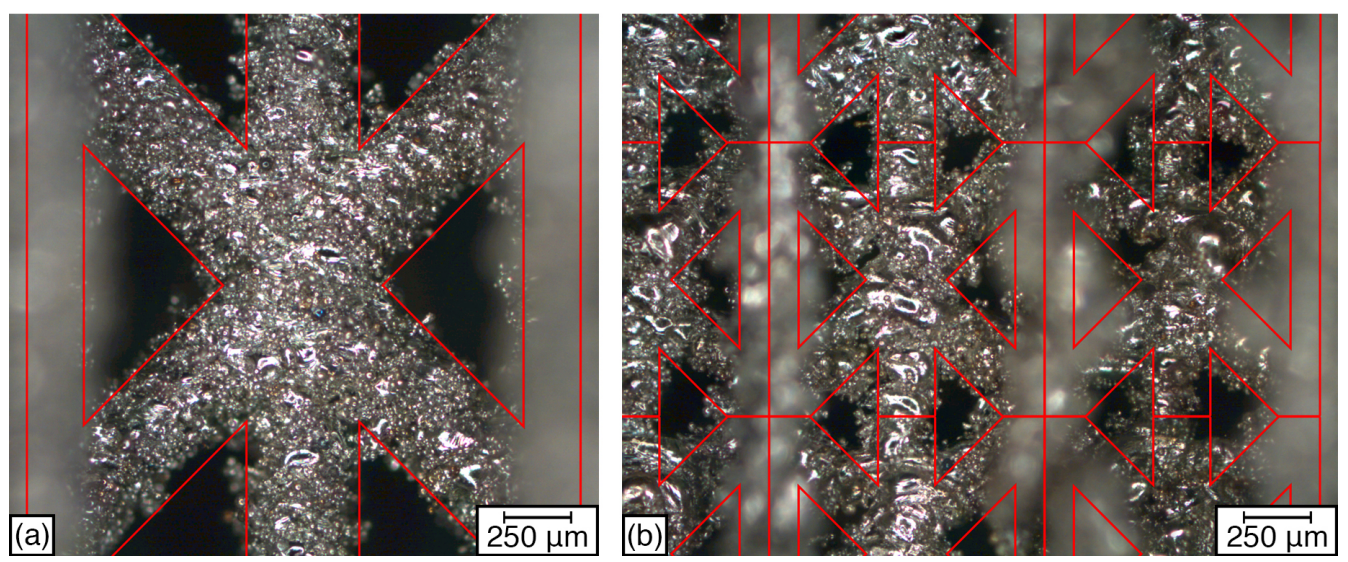

Figure 5: Optical micrograph of an untested (a) LW-SHC and (b) FLW-SHC specimen with nominal geometry bounds overlaid.

The resolution of the geometry of the miniaturised FLW-SHC is, however, less successful: Figure 5 (b). Despite the strut diameter of the FLW-SHC specimen being double the laser spot size, it is clear that the features were too small to accurately replicate (the nominal bounds here being a strut diameter of $200 \mu \mathrm{m}$ ). The melt pool size is expected to be larger than the laser spot size due to the residual heat from previous and adjacent laser pulses combined with the poor pathway for heat removal due to the narrow struts (note that the thermal conductivity of the powder is significantly less than that of the solid [42]). The excess material on the struts in the FLW-SHC case is likely to affect the relative density, and the onset of densification of the lattice in compression. 
The SHC showed consistently accurate agreement with the nominal (CAD) geometry; the surfaces showed less roughness and fewer adhered excess powder particles. The solid walls of the SHC provide much better pathways for heat removal which, in contrast to the FLW-SHC, could help to explain the superior geometric accuracy of the SHC.

\subsection{Macro-scale}

The densities $\rho$ of the as-manufactured cellular materials were obtained from the weight of the test specimens and the volume of the enclosing cylindrical envelope. The relative density was then calculated as described in Section 2.2 The as-manufactured and nominal relative density values (as defined above) are quoted in Table 1 for comparison. The as-manufactured solid-walled SHC specimen has a relative density $1 \%$ higher than the nominal value, confirming that the SLM process has reproduced this relatively simple geometry accurately. The as-manufactured LW-SHC specimen has a relative density 5\% lower than the nominal value. Figure 5(a) shows that the manufactured strut diameters are close to the design value, despite some excess powder particles adhering to the surface. Close inspection of the specimens indicates that the reduced weight may be attributed to poor rendition of the walls along the edges of the specimen, visible on the left of Figure 2 a), which are affected by the lattice trimming method used to fit square unit cells within a circular cylindrical envelope [33]. The small-celled FLW-SHC showed the largest discrepancy in relative density, the as-manufactured specimen being $24 \%$ more dense than the nominal value. This is consistent with the poor resolution of the lattice bars shown in Figure 5 (b), with bar diameters significantly exceeding the nominal values.

\section{Quasi-static mechanical properties}

\subsection{Characterisation of SLM processed 316L stainless steel}

Stainless steel 316L is attractive for this application due to its high strength and strain hardening characteristics, and its conduciveness to additive manufacturing. Laser processing of this material has an additional benefit of grain size reduction, and the formation of fine scale subgrain solidification structures as detailed above, leading to Hall-Petch strengthening [25, 43, 44]. A standard tensile dogbone specimen with a gauge section of width $3 \mathrm{~mm}$, length $20 \mathrm{~mm}$ and thickness $1 \mathrm{~mm}$ was manufactured using the same SLM process as the cellular specimens. The build direction of the tensile specimen (the direction of laser heat input) was perpendicular to the dogbone's loading direction; i.e. the dogbone was lying in the build plane. As noted above, at a microstrucutural level, melt pool boundaries appear elongated perpendicular to the build direction, and will therefore be elongated in the plane of loading of the dogbone specimen. The specimen was tested using a screw-driven materials testing machine, at a nominal strain rate of $10^{-3}$ $\mathrm{s}^{-1}$. Deformation of the gauge section was measured using a laser extensometer. The stress strain curve from this test is shown in Figure 6. Tensile necking was observed to initiate in the specimen at a true strain of 14.5\%, prior to which deformation of the gauge section was uniform. Note that uniform deformation and volume conservation were assumed in the calculation of true stress and strain in Figure 6, and so these quantities are only accurate to the onset of localisation. The $0.2 \%$ offset yield stress was measured to be $580 \mathrm{MPa}$. This can be compared to an AISI annealed value of $235 \mathrm{MPa}$ [35]. To verify this high yield strength, the Vickers hardness was measured prior to tensile testing, and found to be $2.7 \mathrm{GPa}$ (compared to an AISI annealed value of $1.5 \mathrm{GPa}[35]$ ). Both strength and hardness 
measurements indicate process-related property enhancement. Based on the Hall-Petch effect alone, we might expect a yield strength of around 70-80\% of the measured value [43,44]. The remainder is likely due to the aforementioned subgrain solidification structures. Note that microscopy was used to verify that the microstructure of the dogbone test specimen is comparable to that of the AM lattice structures: Figure 3(d)-(f).

The following strain hardening models were fitted to the experimental measurements of true stress $\left(\sigma_{t}\right)$ and logarithmic plastic strain $\left(\varepsilon_{p}\right)$, with $n_{i}$ and $C_{i}$ as fitting parameters. The best fit parameters for each model are quoted in Table 2.

$$
\begin{aligned}
& \text { Hollomon [45]: } \sigma_{t}=C_{1} \varepsilon_{p}^{n_{1}} \\
& \text { Ludwik [46]: } \sigma_{t}=C_{2}+C_{1} \varepsilon_{p}^{n_{1}} \\
& \text { Voce [47]: } \sigma_{t}=C_{2}-\left(C_{2}-C_{1}\right) \exp \left(-n_{1} \varepsilon_{p}\right) \\
& \text { Ludwigson [48]: } \sigma_{t}=C_{1} \varepsilon_{p}^{n_{1}}+\exp \left(C_{2}+n_{2} \varepsilon_{p}\right)
\end{aligned}
$$

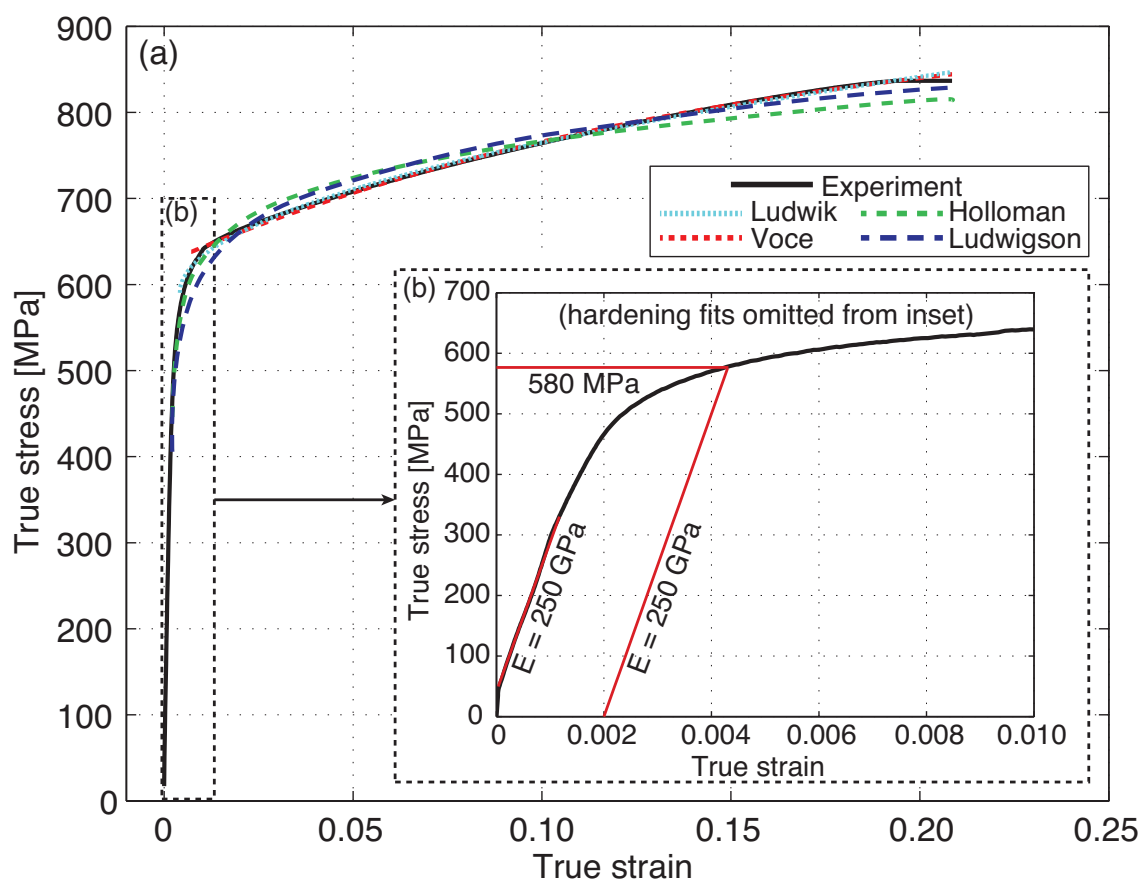

Figure 6: (a) Tensile stress-strain response of a 316L dogbone specimen produced by SLM, with alternative models for the strain hardening superimposed. (b) a magnification of the curve at the onset of yielding.

\subsection{Quasi-static compression of the cellular materials}

One specimen of each geometry was tested in out-of-plane compression at a nominal strain rate of $10^{-3} \mathrm{~s}^{-1}$. Platen displacement was measured with a laser extensometer, with a clip-gauge used to confirm the small strain readings. 


\begin{tabular}{l|ccccc} 
& $C_{1}$ & $n_{1}$ & $C_{2}$ & $n_{2}$ & $R^{2}$ \\
\hline Hollomon (Eq. 11) & $918 \mathrm{MPa}$ & 0.0782 & - & - & $93.7 \%$ \\
Ludwik (Eq. 2] & $578 \mathrm{MPa}$ & 0.5082 & $589 \mathrm{MPa}$ & - & $99.8 \%$ \\
Voce (Eq. 35 & $630 \mathrm{MPa}$ & 6.761 & $914 \mathrm{MPa}$ & - & $99.7 \%$ \\
Ludwigson (Eq. 4) & $957 \mathrm{MPa}$ & 0.0928 & 9.997 & -109.7 & $96.4 \%$
\end{tabular}

Table 2: Fit parameters for strain hardening models.

The nominal compressive strain is defined as $\varepsilon=u / H$, where $u$ is the relative platen displacement, and $H$ the original specimen height. We define the nominal compressive stress to be

$$
\sigma=\frac{4 F}{\pi D^{2}}
$$

where $F$ is the force obtained from the test machine load cell, and $D$ the original specimen diameter. The stress can be expressed in the normalised form

$$
\bar{\sigma}=\frac{\sigma}{\bar{\rho} \sigma_{y}},
$$

where $\bar{\rho}$ is the measured relative density of the specimen, and $\sigma_{y}=580 \mathrm{MPa}$ is the yield stress of the SLM processed 316L stainless steel (the cell wall material). Note that this normalisation has a convenient physical basis, in that the quasi-static out-of-plane compressive yield strength of a square honeycomb (the benchmark cellular structure) is $\sim \bar{\rho} \sigma_{y}[32]$.

The measured compressive responses are shown in Figure 7 a), and with the normalised stress measure in Figure 7(b). The SHC exhibits a nearly constant plateau stress during compression, with a strength $\sim \bar{\rho} \sigma_{y}$, consistent with the relatively modest strain hardening of the alloy [32]. The honeycomb shows negligible softening, indicating limited buckling takes place - a consequence of the relative density and cell aspect ratio [32]. The cell geometry, and hence the regime of collapse, is a consequence of the experimental and manufacturing constraints, as outlined in Section 2.2 The lowest strength configuration is the LW-SHC. The FLW-SHC shows a slightly higher strength than the LW-SHC, which can be attributed to the discrepancy between the nominal and as-manufactured specimen density and strut geometry at this scale, as described above. However, when normalised by relative density, the strengths of the LW-SHC and FLW-SHC are similar. The strengthening effect of the excess strut width and adhered powder particles in the FLWSHC case therefore appears to scale with the increase in mass. Based on the model of [1, 2], the compressive strength of an open-cell foam would be $\bar{\sigma} \approx 0.3 \bar{\rho}^{1 / 2}$, which is marked on Figure 7 (b) for comparison (taking $\bar{\rho}=0.21$ ). This indicates that the lattice-walled structures have an out-of plane compressive strength approximately halfway between that of a foam and a square honeycomb of the same relative density. Comparison with the results of [18] show that their normalised compressive strengths are comparable to lattices such as the pyramidal truss (though these differ in their post-buckling response). Like the SHC specimen, both lattice structures show no softening on compression (and hence no sign of macroscopic buckling), and instead progressively strengthen. The FLW-SHC exhibits a higher degree of strengthening during compression compared to the LW-SHC, which may be a consequence of the lower porosity of 
the cell walls (Figure 5) resulting from the poor geometry resolution.

Macro photographs of the collapse progression at specific strains are shown in Figure 8 For all three cases, the visible cell walls (i.e. those in the plane of the photograph in Figure 8) show little sign of buckling. It can therefore be expected that the majority of cell walls in the specimen interior also deform without significant buckling occurring. This is consistent with the lack of softening observed in the measured compressive stress-strain responses, as noted above. The exception to this is the unconstrained edges of the cell walls on the perimeter of the square honeycomb; however, these are not expected to contribute significantly to the overall strength.
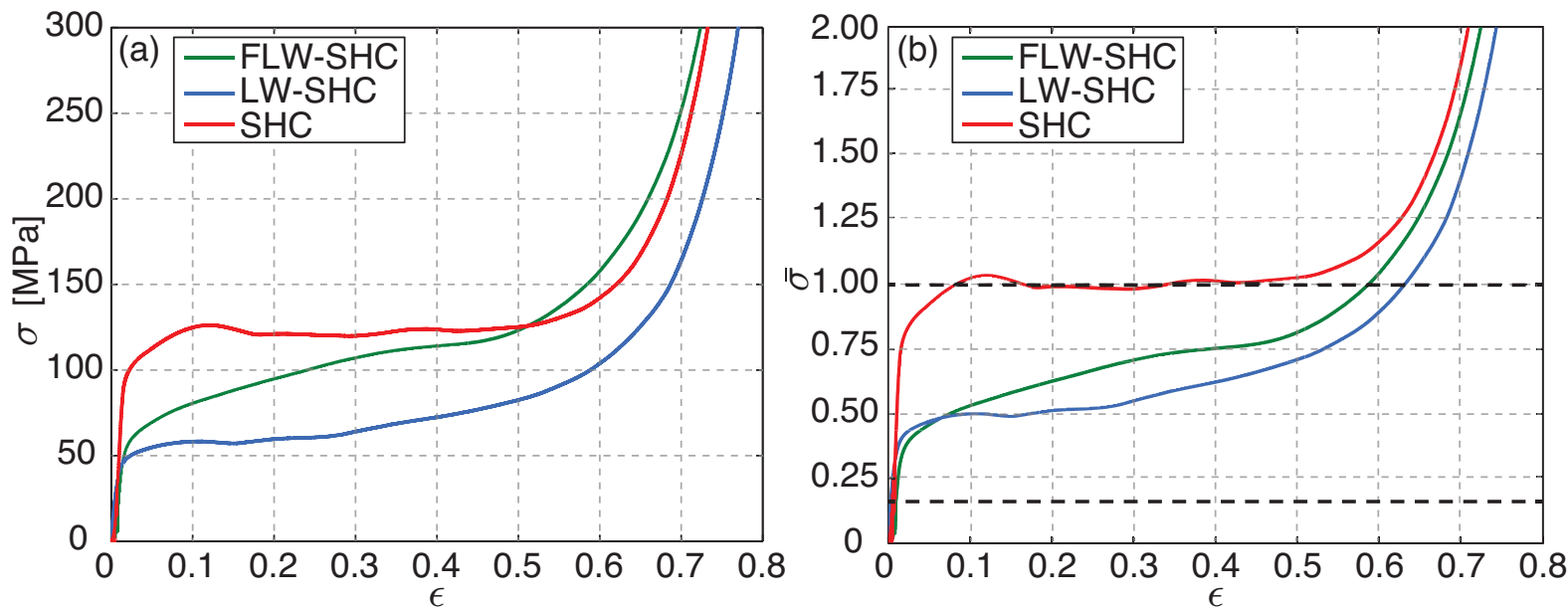

Figure 7: (a) Nominal compressive stress-strain and (b) normalised compressive stress-strain response of specimens. The upper dashed line in (b) represents a collapse strength of $\sim \bar{\rho} \sigma_{y}$ and the lower line represents that of an open-cell foam of the same cell wall material [1] 2].

\subsection{Discussion}

As noted in the introduction, Calladine and English [10] discuss how the characteristics of the quasi-static compressive response of a structure can provide an insight into its dynamic response. All three structures here collapse with a plateau-like stress-strain curve (i.e. they are a 'Type I' response, as defined by these authors). This would indicate a lower sensitivity to inertial effects during dynamic collapse compared to a structure which shows stronger buckling-related softening (i.e. a 'Type II' response). However, the SHC specimen does exhibit a small degree of softening after reaching its initial peak strength at $\varepsilon \approx 0.12$ as shown in Figure 7 This suggests that, of the three, the SHC may be marginally more susceptible to inertial stabilisation.

Next, the quasi-static performance of these AM cellular materials are compared to alternative cellular structures in the literature. Consider the energy absorbed by the specimen to be

$$
W\left(\varepsilon_{1}\right)=\int_{0}^{\varepsilon_{1}} \sigma d \varepsilon
$$




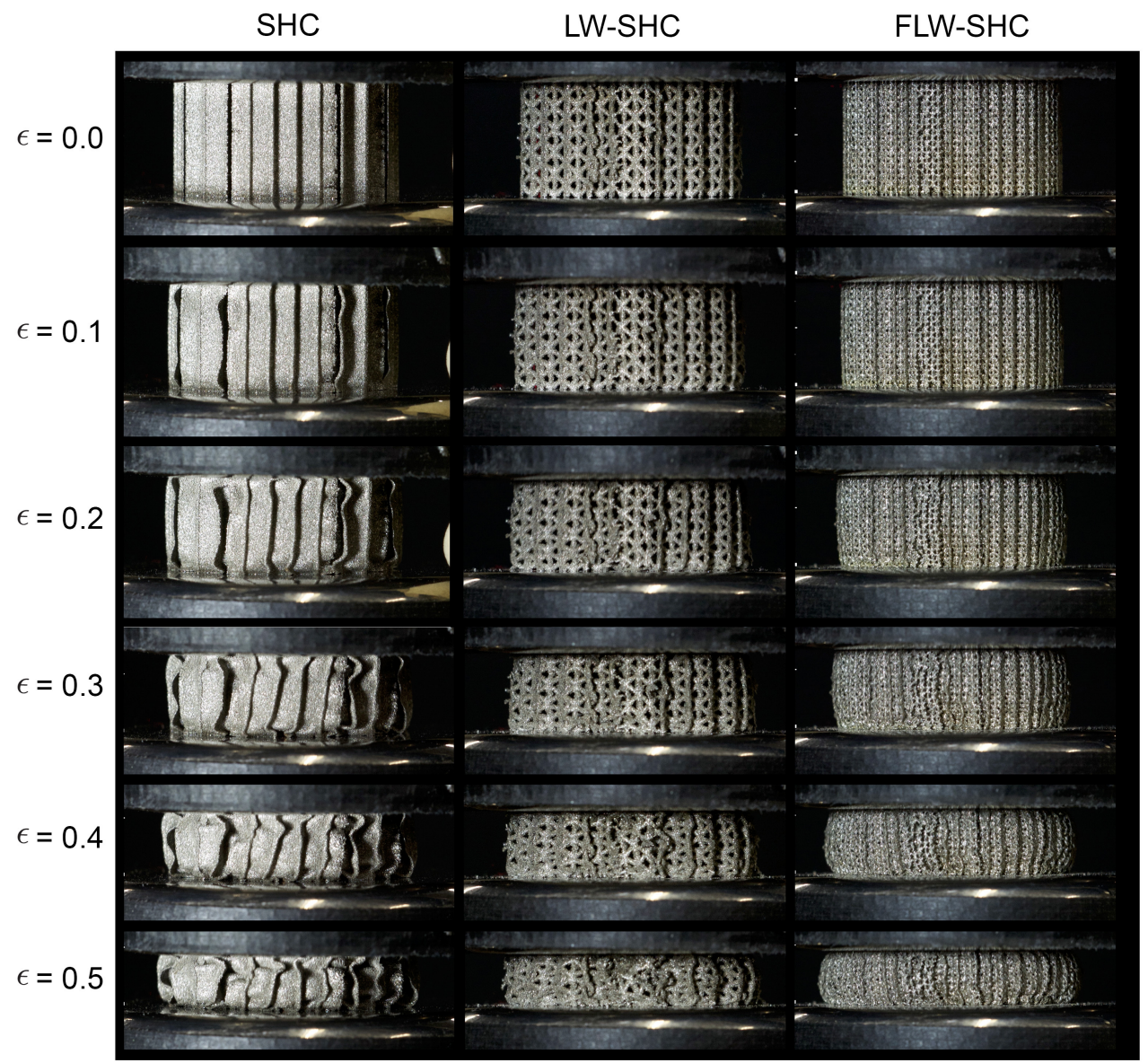

Figure 8: Photographs of the quasi-static compressive collapse of the three cellular specimen geometries, at the nominal strain values indicated.

where $\sigma$ and $\varepsilon$ are the nominal compressive stress and strain, respectively. This can be mass normalised as follows

$$
\bar{W}\left(\varepsilon_{1}\right)=\frac{W\left(\varepsilon_{1}\right)}{\bar{\rho} \rho_{s}} .
$$

Figure 9 shows the specific energy absorption evaluated to $\varepsilon_{1}=0.50$ for the three AM cellular materials. This is plotted against the normalised initial peak stress $\left(\bar{\sigma}_{p}\right)$. For specimens that exhibit softening on plastic collapse (i.e. a Type II structure), $\bar{\sigma}_{p}$ is taken to be the maximum normalised stress recorded before the onset of softening. For specimens that show a plateau in strength or continual strain hardening on plastic collapse (i.e. a Type I structure), $\bar{\sigma}_{p}$ is taken to be the $0.2 \%$ offset yield stress. For comparison, we have included in the Figure published experimental results for some alternative cellular materials:

- Additively manufactured metallic lattice materials, including the 316L stainless steel BCC and BCC-Z lattices produced using SLM, reported by [7, 24].

- Low relative density square honeycombs $(\bar{\rho}=0.10)$ fabricated from 304 stainless steel, tested by [31].

- Cellular structures assembled from square tubes of aluminium alloy 6061-T6, tested by [49]. 
- Metallic foams, produced from various aluminium alloys, as reported by [2].

The lattice-walled structures, the LW-SHC and FLW-SHC, perform similarly to the higher relative density metal foams, and the cellular structures of [49]. They are comparable in terms of normalised peak stress with the additively manufactured BCC and BCC-Z lattices of [7, 24], but show a higher normalised energy absorption. The SHC specimen from the current investigation has a higher normalised peak stress and energy absorption than the lattice materials and foams. The square honeycomb specimen of [31] exhibited similar specific energy absorption to this, but a much higher normalised peak stress, probably due to its strong softening in compression, as a result of cell wall buckling and the tearing of brazed joints [31].

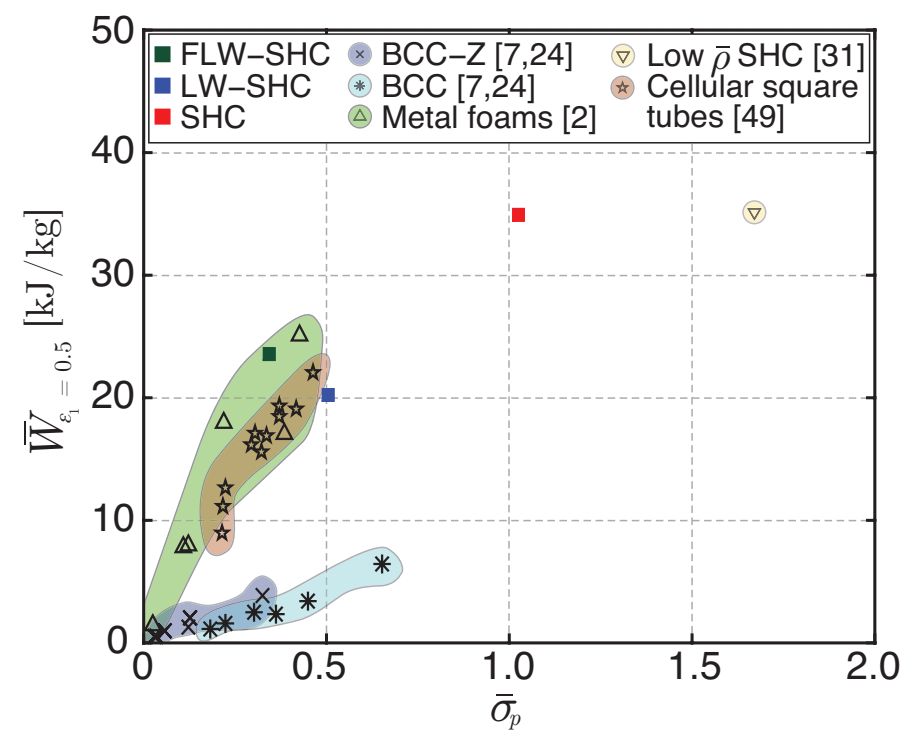

Figure 9: Quasi-static specific energy absorption to 50\% nominal compressive strain vs normalised initial peak stress, as defined in the text. A comparison is shown with alternative cellular structures in the literature.

\section{Dynamic compression}

Dynamic compression of the cellular specimens was carried out using a maraging steel Hopkinson bar apparatus, illustrated in Figure 10. Two test configurations were considered, enabling measurement of the transient forces acting on either side of the specimen with respect to the impacted face:

(i) In the first configuration, Figure 10 a), the cellular specimen is mounted to the Hopkinson bar using a thin layer of two-sided adhesive tape. A steel projectile of mass $0.100 \mathrm{~kg}$ is fired at the specimen using a gas gun apparatus. The time history of the total force acting on the distal face of the specimen is then measured via a pair of strain gauges mounted diametrically opposite each other on the Hopkinson bar at a distance of $285 \mathrm{~mm}$ (ten bar diameters) from the impacted end. These stress measurements are subsequently denoted 'distal face'.

(ii) In the second configuration, Figure 10 (b), the cellular specimen is mounted onto the $0.100 \mathrm{~kg}$ projectile, and both are impacted into the Hopkinson bar using the gas gun apparatus. Note that the masses of the specimens are of 
(a) Distal Face

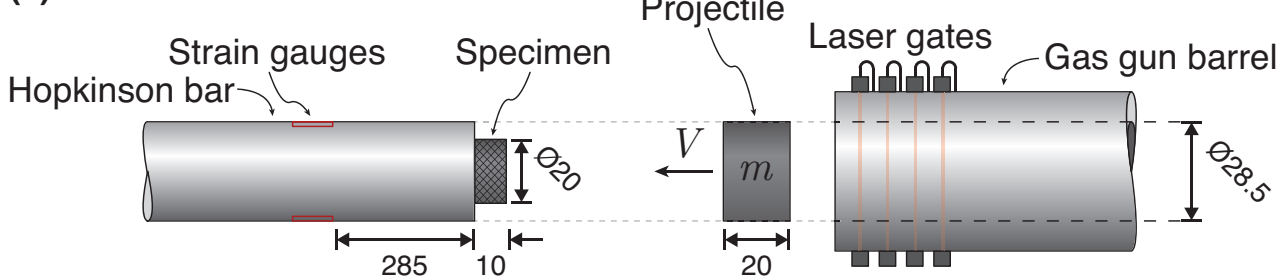

(b) Impacted Face

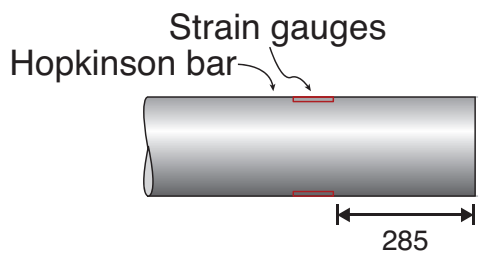
on the Hopkinson bar is again recorded via the pair of strain gauges, and is subsequently denoted the 'impacted face' measurement.

20

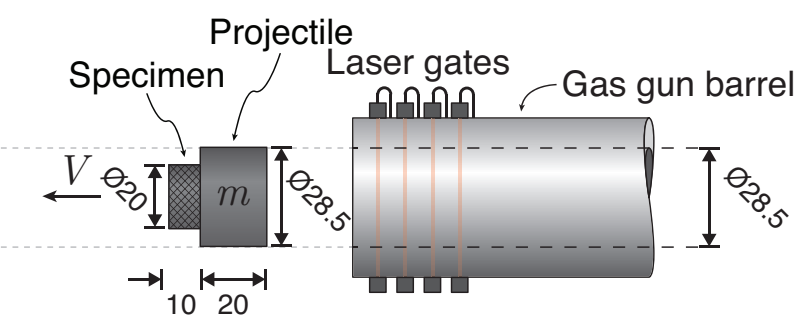

the order $0.005 \mathrm{~kg}$, and therefore small with respect to the projectile mass. The time history of the force acting

Not to scale, all dimensions in $\mathrm{mm}$

Figure 10: Schematic of Hopkinson bar apparatus in (a) distal face and (b) impacted face configurations

Specimens were tested at impact velocities of 50,100 and $150 \mathrm{~ms}^{-1}$, in both distal face and impacted face configurations. These velocities correspond to initial nominal strain rates of approximately $5 \times 10^{3}, 10 \times 10^{3}$ and $15 \times 10^{3}$ $\mathrm{s}^{-1}$, respectively. Hopkinson bar data was corrected for wave dispersion using the Fast Fourier Transform method described in [50, 51] for tests at 50 and $100 \mathrm{~ms}^{-1}$ (this was not possible for the $150 \mathrm{~ms}^{-1}$ impacts due to frequencies exceeding the limits of the dispersion correction process). For reference, the specimen identification numbers, properties and testing conditions for all experiments referred to subsequently are tabulated in Appendix A

\subsection{Dynamic stress-strain results}

To support physical interpretation of the results, the force-time history obtained from the Hopkinson bar was converted into a dynamic stress-strain measurement as follows. The time history of the nominal compressive strain of the cellular specimens, $\varepsilon(t)$, is obtained using high speed photography, using successive images to track the projectile's leading face. The nominal strain is given by the change in the separation of the faces of the projectile and Hopkinson bar, divided by the initial specimen height, $H$. Note that direct measurement of the nominal strain from high speed photography was necessary, due to deceleration of the projectile during the impact. For reference, the projectile velocity variation during specimen deformation for different specimens and impact velocities are given in Appendix B The nominal stress is obtained at either the impacted or distal face, by converting the transient force measurement using Equation 5, and normalising as given in Equation 6 


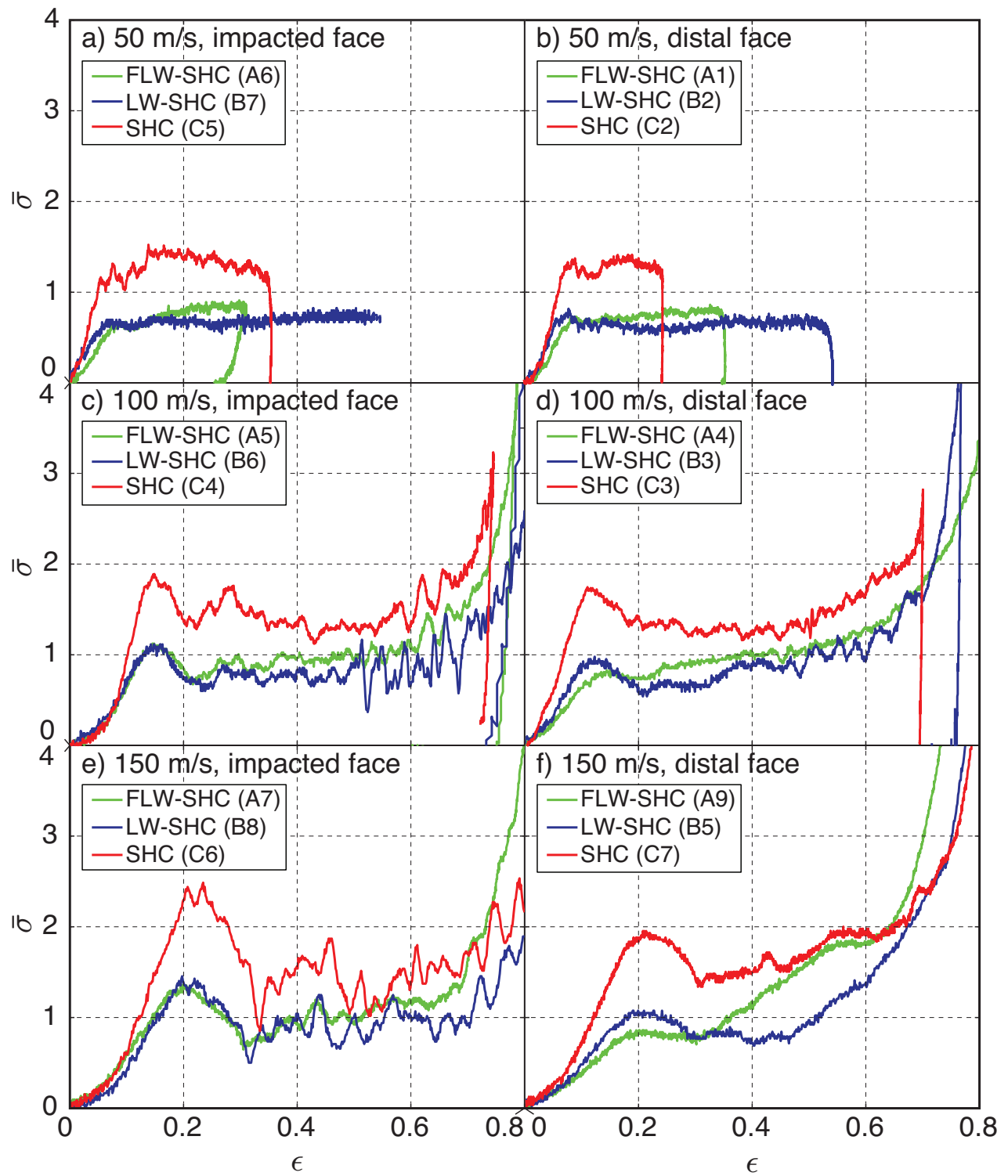

Figure 11: Normalised stress (obtained from the Hopkinson bar) vs. nominal strain (measured using high speed photography) for the impact experiments. The specimen ID labels are quoted in the legend; further data for each can be found in Appendix A 
The results are shown in Figure 11 for the three specimen types impacted at three different velocities. For tests conducted at $100 \mathrm{~ms}^{-1}$ and $150 \mathrm{~ms}^{-1}$, all specimens show a rise in stress to an initial peak, followed by a plateau phase, and finally a steep rise in stress at the onset of densification. For impacts at $50 \mathrm{~ms}^{-1}$, only the plateau phase is captured, due to deceleration of the projectile (see Appendix B. As the impact velocity is increased, more pronounced oscillations in the measured stress are seen for the impacted face configuration, as predicted in [34]. In the distal face configuration, these are dampened as stress waves propagate through the specimen before reaching the Hopkinson bar.

The dynamic compressive responses of the three cellular materials show similar trends to the quasi-static case: the SHC specimen has a higher compressive strength than the lattice configurations, and the two lattice cases show a similar response once normalised by relative density (Figure 11]. However, the dynamic compressive strengths of all three materials exceed the quasi-static cases, and increase with impact velocity. Three factors may contribute to this dynamic strength elevation [31]: the strain rate sensitivity of the 316L stainless steel; inertial stabilisation of buckling phenomena; and wave propagation effects, i.e. the concentration of plastic deformation near the impacted face. As the impact velocity is increased, the initial peaks in stress measured at the impacted face begin to exceed those in the distal face configuration. The effect is marginal at $100 \mathrm{~ms}^{-1}$, but becomes more pronounced at $150 \mathrm{~ms}^{-1}$. This is an indication of wave propagation effects at these impact velocities, and is consistent with the observations of [31] for the onset of this regime. All three specimens, but the SHC in particular, show an increase in the degree of softening after the initial peak strength, compared to the quasi-static case. This can be attributed to the stabilising effect of lateral inertia, increasing this initial peak in strength relative to the subsequent plateau phase of collapse.

\subsection{Peak stress: impact velocity dependence}

The variation in the initial peak in the normalised stress $\left(\bar{\sigma}_{p}\right.$, defined as the maximum value before the onset of the plateau phase) with impact velocity is shown in Figure $\left.12\right|^{2}$. The peak stresses measured at the impacted face show an approximately linear dependence on impact velocity, for all three cellular materials. The peak stress at the distal face matches this trend up to around $100 \mathrm{~ms}^{-1}$, with the subsequent divergence consistent with the emergence of wave propagation effects. The model of Reid and Peng [52] for plastic wave propagation in a cellular material assumes densification occurs behind the wave front, and predicts a quadratic dependence on impact velocity of the stress at the impacted face:

$$
\bar{\sigma}_{p}=\bar{\sigma}_{p 0}+\frac{\rho_{s} V_{i}^{2}}{\sigma_{y} \varepsilon_{d}}
$$

where $\bar{\sigma}_{p 0}$ is the quasi-static normalised compressive strength, $\varepsilon_{d}$ the densification strain, and $\rho_{s}$ and $\sigma_{y}$ the density and yield strength of the cell wall material, respectively. The linear trends observed here therefore suggest that these experiments lie outside of this regime. Radford et al. [31] proposed small strain, one-dimensional elastic-plastic wave propagation along the cell walls as a model for the impact velocity dependence of the impacted face stress, for a

\footnotetext{
${ }^{2}$ Note that this discussion concerns the initial peak stress, but this is not necessarily the maximum stress observed during compressive collapse up to the point of densification. Some configurations, particularly the lattice-walled cases, show significant strengthening throughout the compression (Figure 11. This additional strengthening will be relevant for subsequent discussions of energy absorbing performance.
} 
stainless steel square honeycomb. This analysis gives:

$$
\bar{\sigma}_{p} \approx 1+\frac{\rho_{s} c_{p l} V_{i}}{\sigma_{y}}
$$

where $\sigma_{y}$ and $c_{p l}$ are the yield strength and plastic wave speed, respectively, of the cell wall material (i.e. the $316 \mathrm{~L}$ stainless steel). This predicts the correct linear trend observed in the experiments. However, the analysis does not account for material strain rate sensitivity (Radford et al. [31] overcome this by selecting a high strain rate value for $\left.\sigma_{y}\right)$. Selecting the measured quasi-static value for $\sigma_{y}$ for the SLM 316L stainless steel, and taking $c_{p l}=\sqrt{E_{t} / \rho} \approx 400$ $\mathrm{ms}^{-1}$ gives $\bar{\sigma}_{p} \approx 1.8$ at $V_{i}=150 \mathrm{~ms}^{-1}$. This under-predicts the measured velocity sensitivity of the SHC specimen. It is a closer match to the lower velocity sensitivity measured for the lattice-walled LW-SHC and FLW-SHC specimens, but over-predicts the magnitude of the stress. These observations indicate that a more detailed analysis is necessary to correctly capture the velocity dependence of the compressive strength in this regime, accounting for material strain rate dependence and the influence of cellular topology on the propagation of plastic deformation.

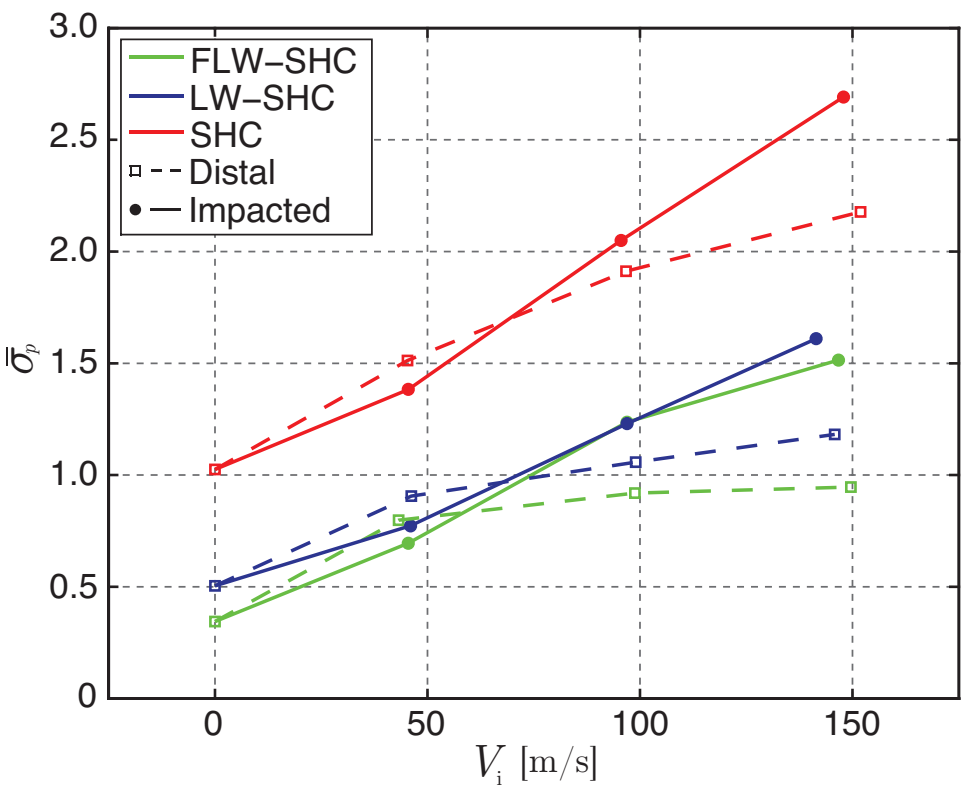

Figure 12: Normalised peak stress vs impact velocity.

\subsection{Specimen deformation}

Photographs at nominal compressive strains of 0.1 and 0.3 are shown in Figure 13 for quasi-static loading and the minimum and maximum impact velocities. For the lattice-walled specimens, the collapse mechanisms appear to remain unchanged with increasing loading rate, as also reported by [7] for BCC and BCC-Z lattices. For the SHC specimen, however, short-wavelength wall buckling is apparent at the higher impact velocity - see the image at $\varepsilon=0.3$ - whereas deformation appears more uniform at the lower loading rates. This is consistent with the increased softening observed in the dynamic compressive stress-strain measurements, Figure 11. A reduction in the buckle wavelength with increasing loading rate can be attributed to inertial stabilisation, and the onset of buckling simultaneously with plastic wave propagation [31, 53]. 


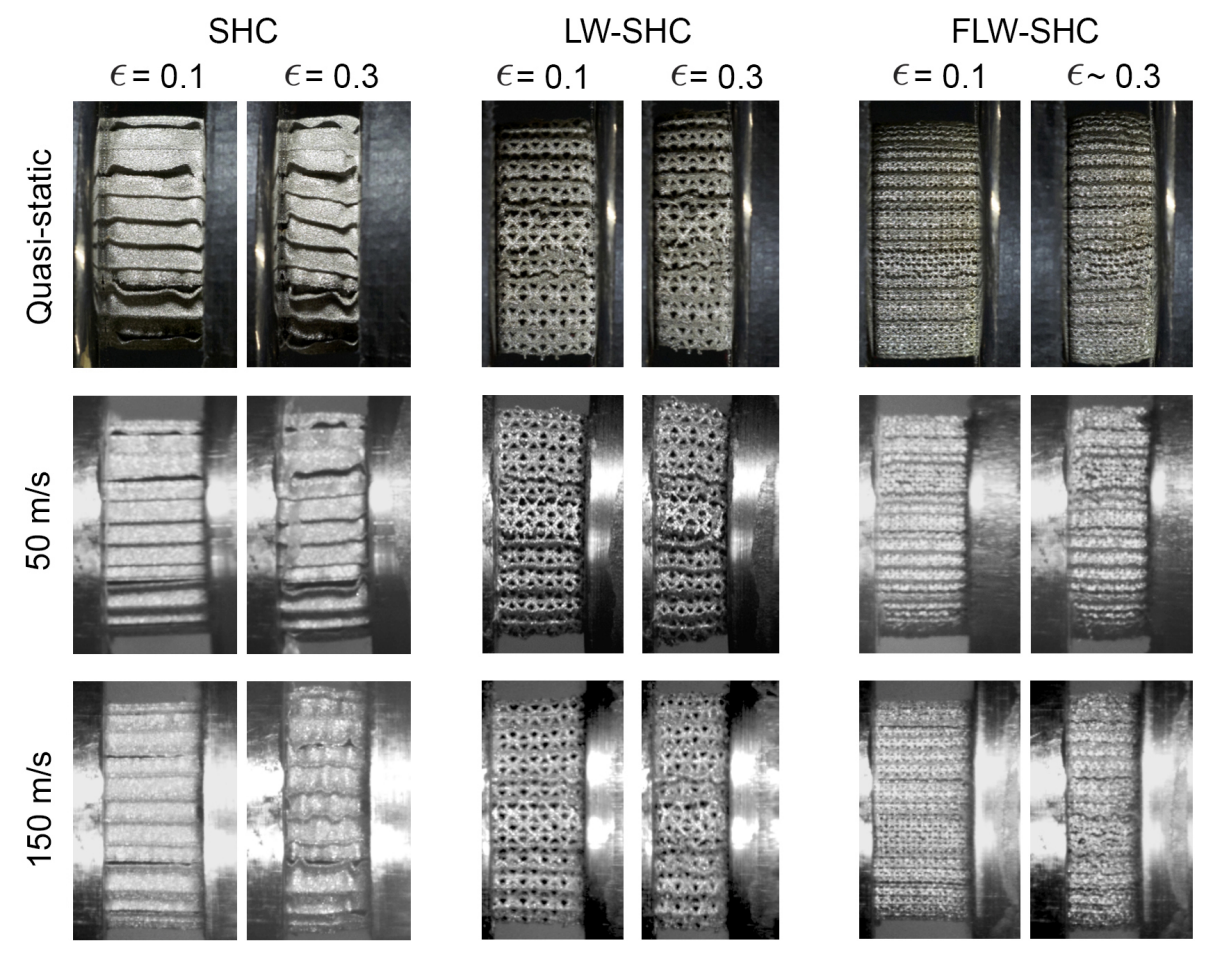

Figure 13: Photographs of different specimens at nominal compressive strains of 0.1 and 0.3 for quasi-static loading and dynamic loading (distal face configuration) at the impact velocities indicated. For the impact cases, the striker is on the right and the Hopkinson bar on the left of the image.

The divergence in the measurements of compressive stress at the impacted and distal faces indicated the emergence of wave propagation effects at the higher impact velocities, and thus the onset of deformation non-uniformity through the height $H$ of the specimen (as defined in Section 2.2). To investigate this further, high speed images were used to track the relative displacements of individual material points through the height $H$ of the specimen during dynamic compression. The FLW-SHC specimen impacted at $150 \mathrm{~ms}^{-1}$ was chosen for analysis, as the lattice structure provided convenient marker points that could be tracked between successive frames (the SHC specimen was unsuitable for this, and the cell size in the coarser LW-SHC was too large for satisfactory results). The results are shown in Figure 14. up to a nominal compressive strain of around 0.40, at which point the deformation of the walls becomes too great for reliable image analysis. The non-uniformity of deformation through the height of the specimen is confirmed, with deformation concentrated at the impacted face. This deformation non-uniformity appears to persist to large nominal compressive strains, and thus the nominal strain is not a precise indication of local deformation conditions at the highest impact velocities. 


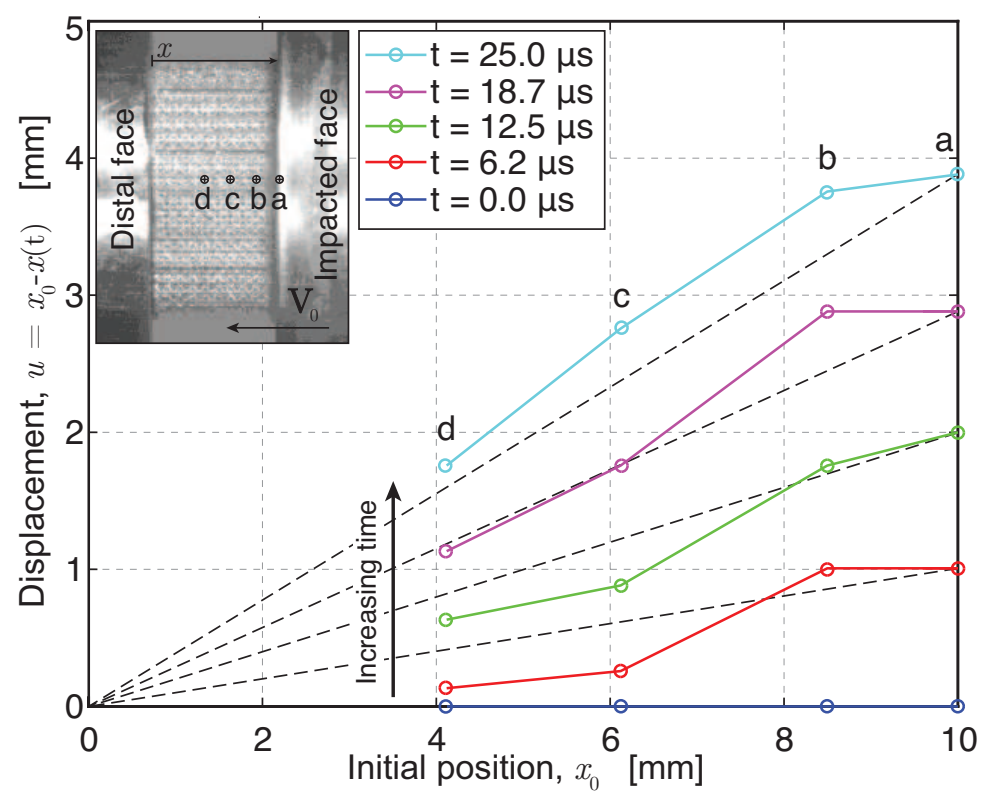

Figure 14: Time evolution of the displacement $u$ of various material points through the height $H$ of the FLW-SHC specimen. The initial position $x_{0}$ (at the time of impact, $t=0$ ) and current position $x(t)$ are measured relative to the distal face. Dashed lines corresponding to uniform deformation are shown for reference. The times shown correspond to nominal compressive strains $\varepsilon=0.10,0.20,0.29$ and 0.38 . The data shown is for an impact velocity of $150 \mathrm{~ms}^{-1}$ in the distal face configuration. Measurement uncertainty was determined to be within $\pm 0.25 \mathrm{~mm}$ upon consideration of video frame rate, projectile velocity, and image resolution.

\section{Energy absorption performance comparison}

We proceed to compare the energy absorption performance of the traditional square honeycomb (SHC) specimen and the hybrid lattice-walled honeycombs proposed as a strategy to support performance optimisation. From an impact mitigation perspective, there are two considerations: the capacity of the structure to absorb energy before the onset of densification, and the peak stresses experienced during this energy absorption phase. To this end, we consider two metrics:

Metric 1 - energy absorption capacity: We opt to take the area under the local stress (measured at the impacted and distal faces) versus nominal strain measurements up to the onset of densification as the first metric (Figure 11):

$$
W\left(\varepsilon_{d}\right)=\int_{0}^{\varepsilon_{d}} \sigma d \varepsilon
$$

where $\varepsilon_{d}$ is the densification strain (obtained using the method defined below). This can be interpreted as the maximum energy absorption per unit volume of cellular material for the cases where uniform deformation is maintained through the height of the specimen during impact (i.e. where the transient stress-time histories at the impacted and distal faces are similar). However, we demonstrate in Appendix C that this metric provides a reasonable indication of the energy 
absorption up to the highest impact velocities considered here, even if this condition is not satisfied 3 , Because, for manufacturing reasons, the specimens compared here vary in relative density, it is also convenient to consider the mass normalised version of this metric:

$$
\bar{W}\left(\varepsilon_{d}\right)=\frac{1}{\bar{\rho} \rho_{s}} \int_{0}^{\varepsilon_{d}} \sigma d \varepsilon
$$

Metric 2 - energy absorption efficiency: Energy absorption efficiency, as referred to by Tan et al. [11] in the context of aluminium alloy foams, considers the ratio of the energy absorbing capacity to the magnitude of the stresses that are experienced. This acknowledges that the maximum stress may be a limiting factor in the specification of an impact energy absorber. Thus, cellular materials that undergo significant strengthening or softening during the plateau phase can be considered a less efficient solution, compared to a perfectly plastic response with the same maximum stress. Here, we define the energy absorption efficiency to be

$$
\eta\left(\varepsilon_{d}\right)=\frac{1}{\sigma_{m}} \int_{0}^{\varepsilon_{d}} \sigma d \varepsilon
$$

where $\sigma_{m}$ is the maximum nominal compressive stress $\sigma$ identified over the range $0 \leq \varepsilon \leq\left.\varepsilon_{d}\right|^{4}$ As above, a normalised form of this metric, $\bar{\eta}=\eta / \bar{\rho}$ allows a fair comparison between the different test specimens.

In order to provide a consistent definition of the densification strain for use in these metrics, we follow the convention of Tan et al. [11] and choose $\varepsilon_{d}$ to be the value of nominal strain that maximises the efficiency, Equation 13
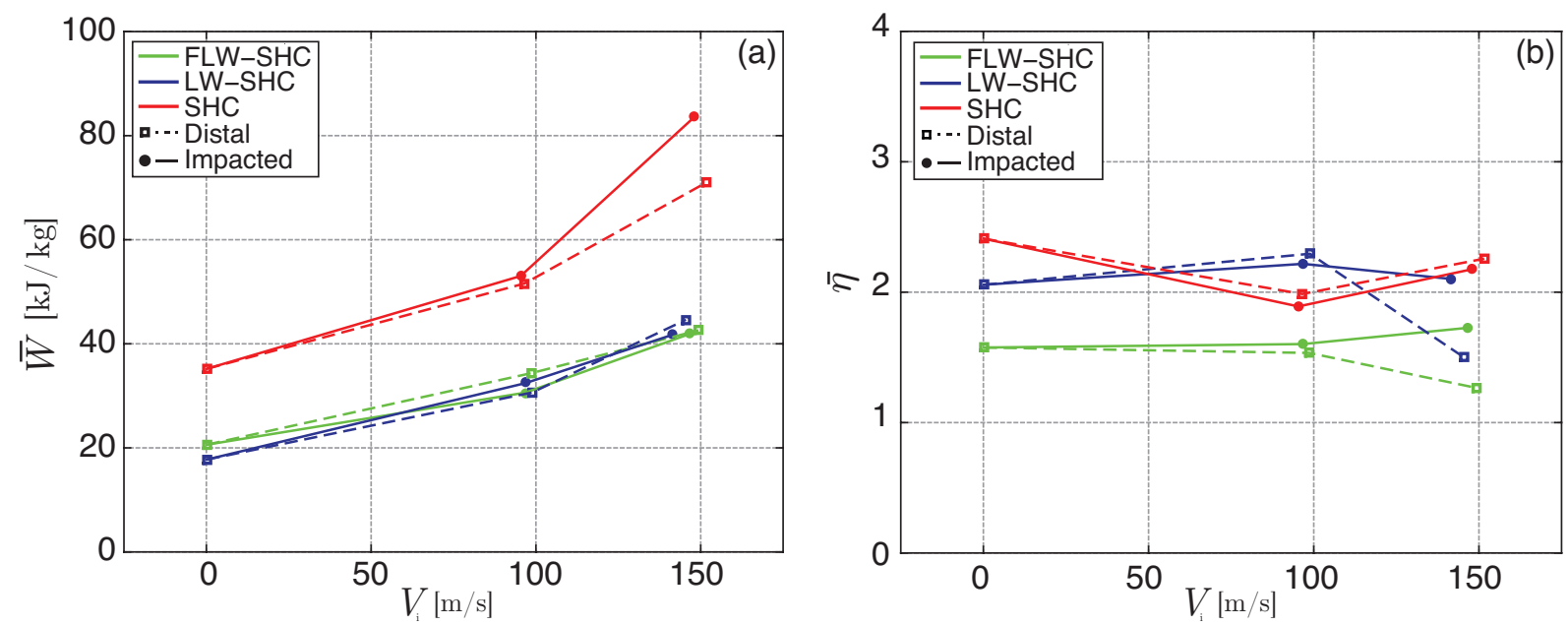

Figure 15: (a) Specific energy absorption and (b) maximum efficiency, vs impact velocity.

\footnotetext{
${ }^{3}$ Energy absorption is commonly evaluated for low velocity impact attenuating materials, whereas impulse-time is preferred at high impact velocities. This investigation is in an intermediate impact velocity regime, but we opt for the former, as it offers advantages in terms of physical insight.

${ }^{4}$ This differs slightly from the definition of [11], in that these authors define $\eta\left(\varepsilon_{1}\right)=\frac{1}{\sigma\left(\varepsilon_{1}\right)} \int_{0}^{\varepsilon_{1}} \sigma d \varepsilon$. By using $\sigma_{m}$ instead of $\sigma\left(\varepsilon_{1}\right)$, the current form seeks to better represent the performance of cellular structures that undergo softening during the plateau phase.
} 
The performance metrics are plotted in Figure 15 for the three cellular materials. Note that results are not shown for $V_{i}=50 \mathrm{~ms}^{-1}$ as the densification strain was not reached in these experiments. At all impact velocities, the SHC shows a higher energy absorption capacity than the two lattice-walled configurations, which both show very similar performance. This is despite the increased softening observed for the SHC specimens at higher impact velocities compared to the lattices (Figure 11), as it is compensated for by the SHC's greater increase in peak strength (Figure 12).

The SHC and LW-SHC specimens are more similar in performance, however, when compared in terms of efficiency. For this metric, the greater degree of softening experienced by the SHC compared to the lattice configurations at higher impact velocities does diminish its performance. At $V_{i}=100 \mathrm{~ms}^{-1}$ the LW-SHC shows the highest efficiency of all three configurations. This indicates that the hybrid solution may be particularly attractive as a basis for performance optimisation in this velocity regime, when the impact velocity is high enough for dynamic buckling effects to diminish the efficiency of the SHC, but before the onset of wave propagation or shock effects. However, the small-celled FLW-SHC shows the lowest efficiency at all impact velocities. This can be attributed to the slightly higher degree of strengthening exhibited by this structure during compression, which in turn may be linked to the higher relative density and poor resolution of the cellular architecture. The AM process limitations therefore must be considered when using this approach to optimise performance.

\section{Optimisation potential}

The hybrid lattice-walled honeycomb structure investigated here was motivated in the introduction as a convenient basis for the optimisation of the geometry for energy absorption. Here, we briefly elaborate on this optimisation potential. By arranging the lattice structure in this way, it is possible to transition smoothly from a lattice to a prismatic honeycomb. It is possible then to systematically investigate whether a geometry of greater energy absorbing efficiency exists between these two extremes for particular loading regimes. The approach is illustrated in Figure 16 On the right of the Figure is shown the hybrid lattice-walled honeycomb structure tested in this study: the solid honeycomb walls have been replaced with a planar truss (similar to the BCC-Z in topology), with bars of circular cross section. The diameter of these bars was chosen such that the lattice has the same relative density as the reference square honeycomb (shown on the left of Figure 16. By then varying the cross-sectional shape of these bars from circular to elliptical, thus reducing the porosity of the walls, while maintaining the same volume fraction of solid, it is possible to evolve the hybrid lattice structure progressively towards the square honeycomb. Thus, it provides a convenient basis structure for exploring local performance optima. This use of hybridisation to enable the systematic evolution of the geometry is an approach to optimisation that is particularly suited to an additive manufacturing route. However, the resolution and geometric accuracy of the AM process will be an important factor in accomplishing this. 


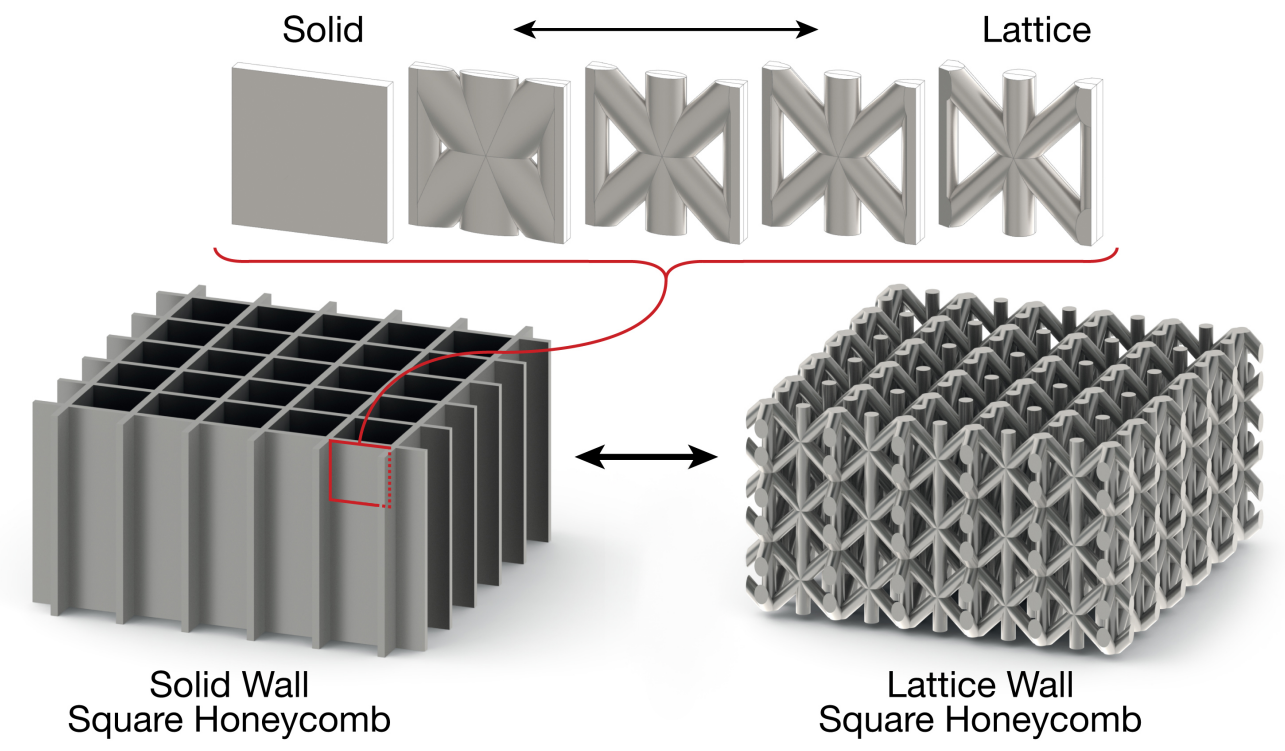

Figure 16: Illustration of the geometry hybridisation concept: replacing the solid walls of a square honeycomb with a lattice truss (at constant relative density), to create a hybrid lattice-walled square honeycomb. This forms a convenient basis for systematically varying the cellular topology between the lattice and square honeycomb extremes, as shown inset, to probe performance optima.

\section{Conclusions}

1. Additive manufacturing, using a Selective Laser Melting (SLM) process, has enabled the successful fabrication of two types of stainless steel cellular materials: a hybrid lattice-walled honeycomb geometry (proposed as a potential optimisation strategy) and a conventional solid-walled square honeycomb.

2. The lattice-walled structures were found to be sensitive to the resolution limitations of the SLM process. As the lattice bar diameter approaches the laser spot size (as is the case for the miniaturised variant, the FLW-SHC), significant errors in the relative density (versus the nominal value) are apparent. The lattice-walled specimens also show defects such as curved vertical bars, which we attribute to the manufacturing process. These sources of geometry errors are likely to be mitigated as additive manufacturing processes advance.

3. The SLM process exposes the material to intense thermal cycles that alter the microstructure, and hence the mechanical performance, compared to conventionally process stainless steel. Hardness and yield strength values were measured that significantly exceed those of bulk annealed 316L stainless steel. The measured grain sizes of 5-15 $\mu \mathrm{m}$ are not small enough to explain this increase in hardness on the basis of Hall-Petch relationships established for this alloy. An additional hardening contribution is therefore attributed to fine-scale sub-grain solidification structures, observed using scanning electron microscopy.

4. Quasi-statically, the lattice-walled honeycombs exhibited greater normalised strength and specific energy absorption than additively manufactured BCC and BCC-Z lattices, reported in the literature. However, they demonstrated lower strength and energy absorption up to the point of densification than similarly-manufactured square honeycombs. 
5. For dynamic deformation, the square honeycomb again demonstrates greater energy absorption up to the point of densification than the lattice-walled structures. However, the initial peak strength of the square honeycomb is more sensitive to the impact velocity than is the case for the lattice specimens. The stabilising effect of lateral inertia during the initial compression of the SHC specimens results in a significant increase in the initial peak strength, particularly at the impacted face.

6. The trade-off between the energy absorption (up to densification) and the maximum stress is encapsulated by the energy absorption efficiency, a metric given by the ratio of these quantities. It is found that, despite its high peak strengths, the SHC specimens demonstrate the greatest efficiency at both the lowest and highest impact velocities (50 and $150 \mathrm{~ms}^{-1}$ ). However, the LW-SHC specimen has a greater efficiency at the intermediate impact velocity $\left(100 \mathrm{~ms}^{-1}\right)$. In this intermediate regime, dynamic buckling effects are present, but wave propagation effects are not yet significant. This indicates that the hybrid lattice-walled solution may have the greatest scope for performance optimisation in this impact velocity range.

7. The miniaturised version of the lattice-walled structure, the FLW-SHC, under-performed compared to the other two structures. This can be attributed to the poor rendition of the geometry and the excess material deposits, increasing the relative density, but without benefiting the mechanical properties. The effects of these deficiencies in build quality were most significant at the intermediate impact velocity $\left(100 \mathrm{~ms}^{-1}\right)$.

8. Divergence of the measured stresses at the impacted and distal faces of the specimens, and identification (via high speed photography) of deformation non-uniformity in the impact direction, indicates that wave propagation effects are beginning to influence the impact response at the maximum impact velocity considered here (150 $\mathrm{ms}^{-1}$ ). Further experiments and numerical analysis will be required to identify how the relative performance of these lattice structures changes as the impact velocity is increased further, up to the regime where wave propagation dominates the response, as reported by [25] for a plate-impact test configuration.

\section{Acknowledgements}

The authors are grateful to AWE for financial support and for providing experimental specimens. We thank M. Cotton, E. Harris, D. Macknelly and M. Swan of AWE for their advice, and assistance with specimen design and manufacture. 


\section{A. Summary of dynamic experimental results}

The following table contains a summary of the experimental results of this study. Energy absorption and maximum efficiency are presented for both measures, for reference: from the projectile kinetic energy change $(U)$ and from the integral of nominal stress vs strain $(W)$ (refer to Appendix C for a discussion of these measures of energy absorption).

The choice of energy absorption measurement method affects the value of the efficiency, but not the overall ranking.

\begin{tabular}{|c|c|c|c|c|c|c|c|c|c|c|c|c|c|}
\hline Specimen & $\bar{\rho}$ & ID & $\begin{array}{c}V \\
{[\mathrm{~m} / \mathrm{s}]}\end{array}$ & Face & $\bar{\sigma}_{p}$ & $\bar{\sigma}_{m}$ & $\begin{array}{c}\bar{U}(\varepsilon=0.5) \\
{[\mathrm{kJ} / \mathrm{kg}]}\end{array}$ & $\begin{array}{c}\bar{W}(\varepsilon=0.5) \\
{[\mathrm{kJ} / \mathrm{kg}]}\end{array}$ & $\varepsilon_{d}$ & $\begin{array}{c}\bar{U}\left(\varepsilon=\varepsilon_{d}\right) \\
{[\mathrm{kJ} / \mathrm{kg}]}\end{array}$ & $\begin{array}{c}\bar{W}\left(\varepsilon=\varepsilon_{d}\right) \\
{[\mathrm{kJ} / \mathrm{kg}]}\end{array}$ & $\bar{\eta}_{U}$ & $\bar{\eta}_{W}$ \\
\hline \multirow[t]{7}{*}{ FLW-SHC } & 0.255 & $\mathrm{~A} 0$ & Q.S. & N/A & 0.34 & 0.93 & N/A & 23.5 & 0.55 & N/A & 26.9 & N/A & 1.57 \\
\hline & 0.255 & A1 & 43.2 & dist. & 0.80 & 0.80 & N/A & N/A & N/A & N/A & N/A & N/A & N/A \\
\hline & 0.258 & A6 & 45.5 & imp. & 0.70 & 0.70 & N/A & N/A & N/A & N/A & N/A & N/A & N/A \\
\hline & 0.257 & A4 & 98.8 & dist. & 0.92 & 1.43 & 33.0 & 30.5 & 0.60 & 39.8 & 40.7 & 1.49 & 1.53 \\
\hline & 0.258 & A5 & 96.9 & imp. & 1.24 & 1.24 & 31.0 & 30.8 & 0.58 & 38.8 & 36.9 & 1.68 & 1.60 \\
\hline & 0.257 & A9 & 149.6 & dist. & 0.95 & 2.09 & 33.7 & 31.9 & 0.63 & 44.5 & 48.9 & 1.14 & 1.26 \\
\hline & 0.255 & A7 & 146.7 & imp. & 1.52 & 1.52 & 24.6 & 34.0 & 0.66 & 45.9 & 48.1 & 1.64 & 1.72 \\
\hline \multirow[t]{7}{*}{ LW-SHC } & 0.195 & B0 & Q.S. & N/A & 0.50 & 0.83 & N/A & 20.3 & 0.57 & N/A & 24.0 & N/A & 2.05 \\
\hline & 0.196 & B2 & 46.2 & dist. & 0.91 & 0.91 & 20.2 & 25.7 & N/A & N/A & N/A & N/A & N/A \\
\hline & 0.194 & B7 & 46.0 & imp. & 0.77 & 0.77 & 19.7 & 25.9 & N/A & N/A & N/A & N/A & N/A \\
\hline & 0.194 & B3 & 99.0 & dist. & 1.06 & 1.15 & 34.9 & 28.7 & 0.60 & 41.2 & 36.9 & 2.56 & 2.29 \\
\hline & 0.195 & B6 & 96.9 & imp. & 1.23 & 1.24 & 27.3 & 28.0 & 0.66 & 41.5 & 38.7 & 2.37 & 2.21 \\
\hline & 0.194 & B5 & 145.8 & dist. & 1.18 & 2.43 & 26.6 & 29.1 & 0.69 & 55.1 & 50.9 & 1.62 & 1.49 \\
\hline & 0.196 & B8 & 141.4 & imp. & 1.61 & 1.61 & 18.8 & 31.6 & 0.72 & 44.3 & 48.0 & 1.94 & 2.10 \\
\hline \multirow[t]{7}{*}{ SHC } & 0.212 & $\mathrm{C} 0$ & Q.S. & N/A & 1.02 & 1.12 & N/A & 34.9 & 0.59 & N/A & 41.5 & N/A & 2.41 \\
\hline & 0.212 & $\mathrm{C} 2$ & 45.3 & dist. & 1.51 & 1.51 & N/A & N/A & N/A & N/A & N/A & N/A & N/A \\
\hline & 0.212 & C5 & 45.5 & imp. & 1.38 & 1.38 & N/A & N/A & N/A & N/A & N/A & N/A & N/A \\
\hline & 0.211 & C3 & 96.7 & dist. & 1.91 & 1.91 & 55.3 & 45.8 & 0.61 & 68.1 & 57.9 & 2.33 & 1.98 \\
\hline & 0.212 & $\mathrm{C} 4$ & 95.5 & imp. & 2.05 & 2.05 & 53.0 & 46.1 & 0.62 & 67.5 & 59.4 & 2.14 & 1.88 \\
\hline & 0.213 & C7 & 151.9 & dist. & 2.18 & 2.22 & 58.4 & 52.7 & 0.66 & 83.3 & 77.3 & 2.42 & 2.25 \\
\hline & 0.212 & C6 & 147.9 & imp. & 2.69 & 2.69 & 34.2 & 55.0 & 0.78 & 81.0 & 89.7 & 1.96 & 2.17 \\
\hline
\end{tabular}

Table 3: Summary of experimental data. Note that the $50 \mathrm{~m} / \mathrm{s}$ tests did not reach full densification, so some metrics are not applicable. 


\section{B. Projectile Velocity Measurements}

Figure 17 shows the variation in striker velocity during the impact of the specimens indicated, obtained from high speed photography. The markers in the figure indicate individual frames from the sequence of high speed images. The variation in velocity is plotted against nominal strain, defined as the change in separation of the faces of the striker and Hopkinson bar, divided by the original height of the specimen, $H$. A second axis shows the striker velocity divided by the original specimen height $H$, which gives the nominal strain rate on impact. For the higher impact velocities, the rate of deformation remains reasonably consistent for the first $50 \%$ nominal compressive strain. The effect of striker deceleration is more severe at an impact velocity of $50 \mathrm{~ms}^{-1}$, and so a constant rate of deformation cannot be assumed in this case.
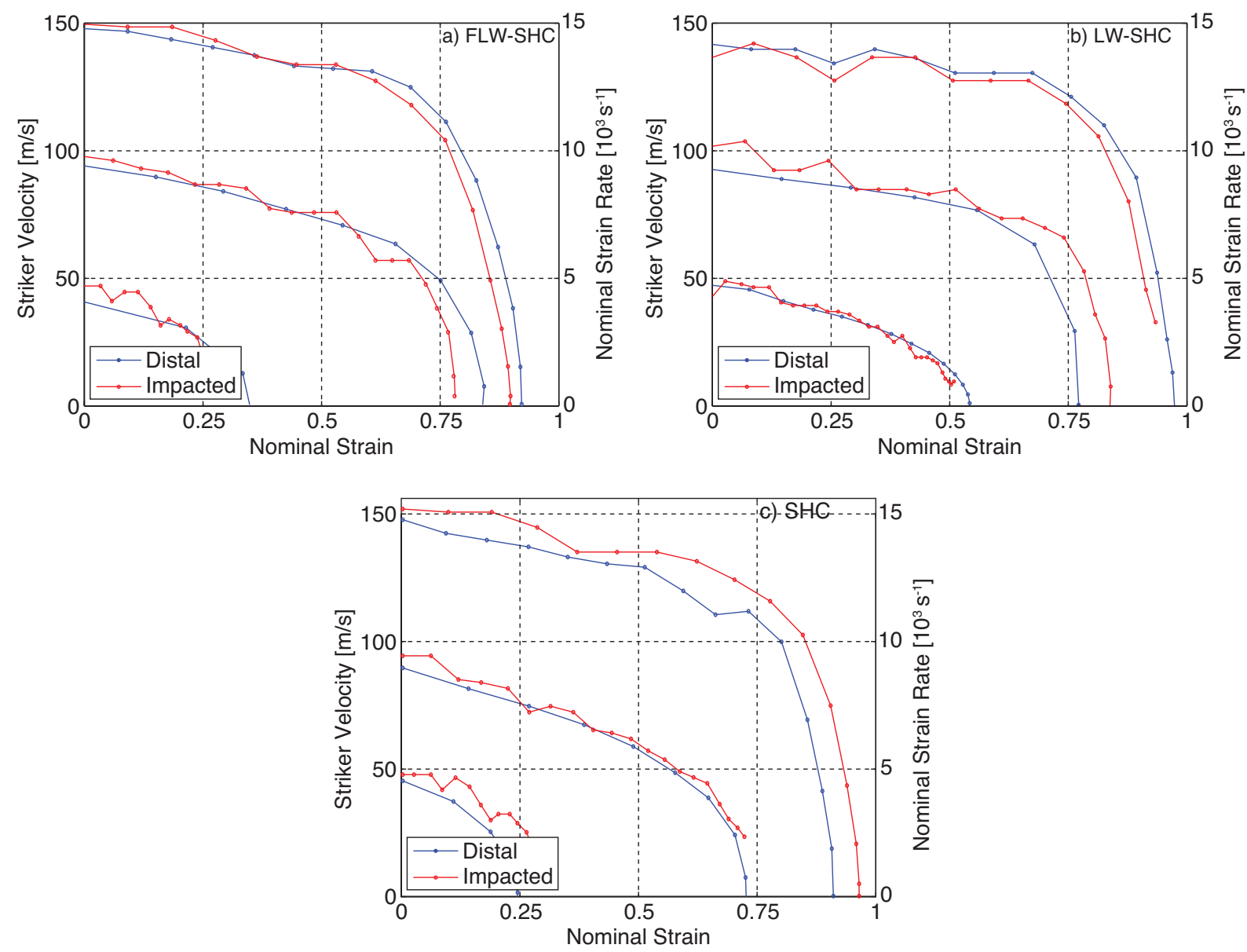

Figure 17: Striker velocity and nominal strain rate vs nominal strain for each of the specimens. 


\section{Energy absorption metric}

If the deformation is uniform through the height $H$ of the specimen, as it is in quasi-static testing, then $W\left(\varepsilon_{d}\right)$ (as defined in Section (6) can be interpreted as the energy absorbed per unit volume of cellular material. As the deformation becomes non-uniform as the impact velocity increases, concentrating at the impacted face, the physical interpretation of this metric is less secure. To assess its validity for these experiments, we consider a second measure of the energy dissipated by the specimen: the drop in kinetic energy of the projectile up to the nominal densification strain, $\varepsilon_{d}$, measured from the high speed photography

$$
U\left(\varepsilon_{d}\right)=\frac{\frac{1}{2} M\left(V_{i}^{2}-V_{d}^{2}\right)}{v_{e}}
$$

where $M$ the projectile mass, $V_{i}$ the impact velocity, $V_{d}$ the measured projectile velocity at $\varepsilon=\varepsilon_{d}$, and the volume of the envelope encapsulating the specimen $v_{e}=\pi D^{2} H / 4$. This can also be presented in a mass-normalised form: $\bar{U}=U /\left(\bar{\rho} \rho_{s}\right)$.

This metric has the advantage of not relying on local stress measurements at the impacted and distal faces, but has the disadvantage of lacking the time-resolution and precision of the data obtained from the Hopkinson bar, due to the limitations of the high speed photography. A comparison between the two energy absorption metrics, $\bar{W}$ and $\bar{U}$, is shown in the following figure. As previously noted, the additional projectile mass in the impacted face configuration tests (due to the mounting of the specimen on the projectile) is less than 5\%. The average difference between the two metrics is 9\% (the full data are presented in Appendix A, but note that there is much more uncertainty in the kinetic energy measurement and this uncertainty grows with impact velocity, as it is associated with the high speed camera frame-rate and resolution. Hence, $\bar{W}$ is selected as the preferred metric.
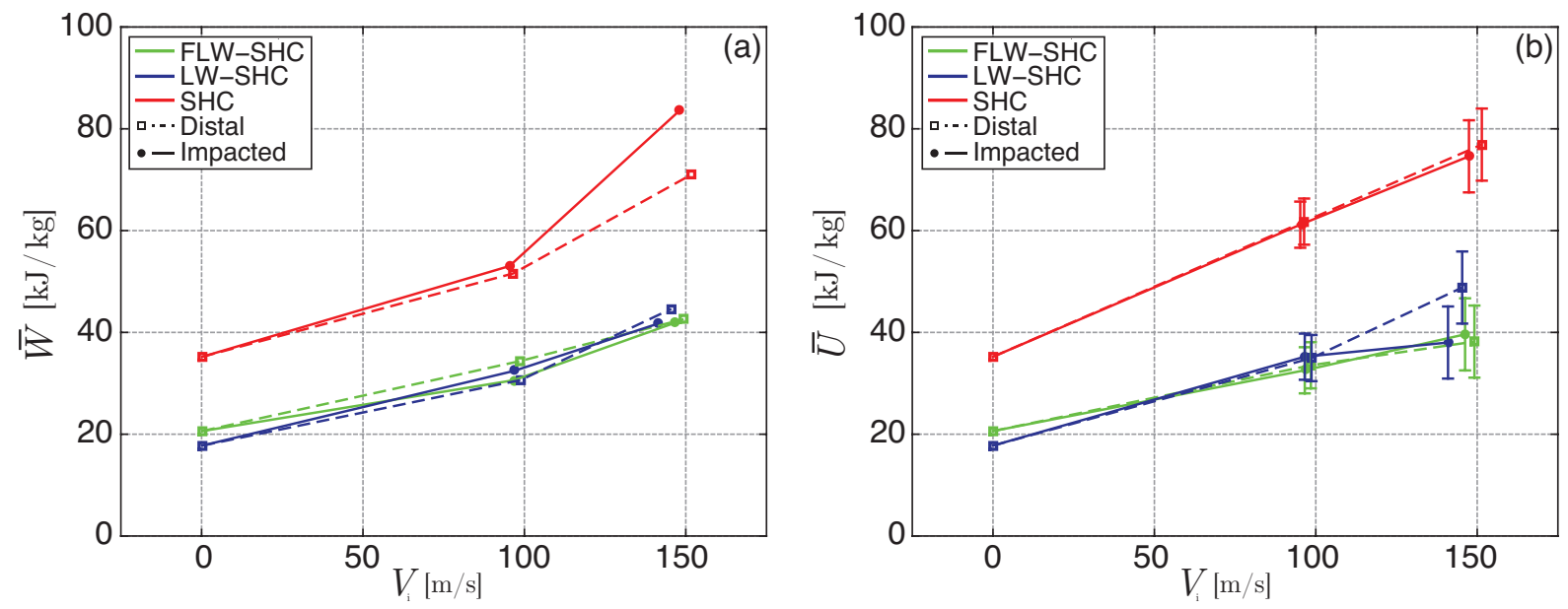

Figure 18: Specific energy absorption vs impact velocity, calculated using (a) the Hopkinson bar local stress measurements and (b) the change in projectile kinetic energy. 


\section{References}

[1] M. F. Ashby, L. Gibson, Cellular solids: Structure and properties, Cambridge University Press, 1999.

[2] M. F. Ashby, A. G. Evans, N. A. Fleck, L. Gibson, J. W. Hutchinson, H. Wadley, Metal Foams - A Design Guide, Butterworth-Heinemann, 2000.

[3] S. Maiti, L. Gibson, M. F. Ashby, Deformation and energy absorption diagrams for cellular solids, Acta Metallurgica 32 (11) (1984) 1963-1975.

[4] K. P. Dharmasena, D. T. Queheillalt, H. Wadley, P. Dudt, Y. Chen, D. Knight, A. G. Evans, V. S. Deshpande, Dynamic compression of metallic sandwich structures during planar impulsive loading in water, European Journal of Mechanics - A/Solids 29 (2010) 56-67.

[5] N. A. Fleck, V. S. Deshpande, The Resistance of Clamped Sandwich Beams to Shock Loading, Journal of Applied Mechanics 71 (3) (2004) 386-401.

[6] V. S. Deshpande, M. F. Ashby, N. A. Fleck, Foam topology bending verseus stretching dominated architectures, Acta Materialia 49 (2001) 1035-1040.

[7] M. Smith, W. Cantwell, Z. Guan, S. Tsopanos, M. Theobald, G. Nurick, G. Langdon, The quasi-static and blast response of steel lattice structures, Journal of Sandwich Structures and Materials 13 (4) (2010) 479-501.

[8] D. D. Radford, G. J. McShane, V. S. Deshpande, N. A. Fleck, The response of clamped sandwich plates with metallic foam cores to simulated blast loading, International Journal of Solids and Structures 43 (7-8) (2006) $2243-2259$.

[9] G. J. McShane, D. D. Radford, V. S. Deshpande, N. A. Fleck, The response of clamped sandwich plates with lattice cores subjected to shock loading, European Journal of Mechanics - A/Solids 25 (2) (2006) 215-229.

[10] C. Calladine, R. English, Strain-Rate and Inertia Effects in the Collapse of Two Types of Energy-Absorbing Structure, International Journal of Mechanical Sciences 26 (11/12) (1984) 689-701.

[11] P. J. Tan, J. J. Harrigan, S. R. Reid, Inertia effects in uniaxial dynamic compression of a closed cell aluminium alloy foam, Materials Science and Technology 18 (5) (2002) 480-488.

[12] S. R. Reid, Plastic deformation mechanisms in axially compressed metal tubes used as impact energy absorbers, International Journal of Mechanical Sciences 35 (2) (1993) 1035-1052.

[13] Z. Xue, J. W. Hutchinson, A comparative study of impulse-resistant metal sandwich plates, International Journal of Impact Engineering 30 (10) (2004) 1283-1305.

[14] N. A. Fleck, V. S. Deshpande, Collapse of truss core sandwich beams in 3-point bending, International Journal of Solids and Structures (2001) 6275-6305. 
[15] V. S. Deshpande, N. A. Fleck, High strain rate compressive behaviour of aluminium alloy foams, International Journal of Impact Engineering 24 (2000) 277-298.

[16] S. Lee, F. Barthelat, J. W. Hutchinson, H. D. Espinosa, Dynamic failure of metallic pyramidal truss core materials Experiments and modeling, International Journal of Plasticity 22 (11) (2006) 2118-2145.

[17] H. Wadley, Fabrication and structural performance of periodic cellular metal sandwich structures, Composites Science and Technology 63 (16) (2003) 2331-2343.

[18] H. N. Wadley, Multifunctional periodic cellular metals, Philos Trans A Math Phys Eng Sci 364 (1838) (2006) $31-68$.

[19] T. A. Schaedler, A. J. Jacobsen, A. Torrents, A. E. Sorensen, J. Lian, J. R. Greer, L. Valdevit, W. B. Carter, Ultralight metallic microlattices, Science 334 (6058) (2011) 962-5.

[20] A. Lausic, C. Steeves, G. Hibbard, Effect of grain size on the optimal architecture of electrodeposited metal/polymer microtrusses, Journal of Sandwich Structures and Materials 16 (3) (2014) 251-271.

[21] L. C. Montemayor, L. R. Meza, J. R. Greer, Design and Fabrication of Hollow Rigid Nanolattices via Two-Photon Lithography, Advanced Engineering Materials 16 (2) (2014) 184-189.

[22] X. Zheng et. al, Ultralight, ultrastiff mechanical metamaterials, Science 344 (6190) (2014) 1373-1377.

[23] C. Yan, L. Hao, A. Hussein, P. Young, D. Raymont, Advanced lightweight 316L stainless steel cellular lattice structures fabricated via selective laser melting, Materials and Design 55 (2014) 533-541.

[24] S. McKown, Y. Shen, W. K. Brookes, C. J. Sutcliffe, W. J. Cantwell, G. S. Langdon, G. N. Nurick, M. D. Theobald, The quasi-static and blast loading response of lattice structures, International Journal of Impact Engineering 35 (8) (2008) 795-810.

[25] R. E. Winter, M. Cotton, E. J. Harris, J. R. Maw, D. J. Chapman, D. E. Eakins, G. McShane, Plate-impact loading of cellular structures formed by selective laser melting, Modelling and Simulation in Materials Science and Engineering 22 (2) (2014) 025021.

[26] R. A. W. Mines, S. Tsopanos, Y. Shen, R. Hasan, S. T. McKown, Drop weight impact behaviour of sandwich panels with metallic micro lattice cores, International Journal of Impact Engineering 60 (2013) 120-132.

[27] R. Gumruk, R. A. W. Mines, Compressive behaviour of stainless steel micro-lattice structures, International Journal of Mechanical Sciences 68 (2013) 125-139.

[28] S. Van Bael, G. Kerckhofs, M. Moesen, G. Pyka, J. Schrooten, J. P. Kruth, Micro-CT-based improvement of geometrical and mechanical controllability of selective laser melted Ti6Al4V porous structures, Materials Science and Engineering: A 528 (24) (2011) 7423-7431. 
[29] F. Brenne, T. Niendorf, H. J. Maier, Additively manufactured cellular structures: Impact of microstructure and local strains on the monotonic and cyclic behavior under uniaxial and bending load, Journal of Materials Processing Technology 213 (9) (2013) 1558-1564.

[30] Z. Ozdemir, E. Hernandez-Nava, A. Tyas, J. A. Warren, S. D. Fay, R. Goodall, I. Todd, H. Askes, Energy absorption in lattice structures in dynamics: experiments, International Journal of Impact Engineering (2015) $49-61$.

[31] D. Radford, G. McShane, V. Deshpande, N. Fleck, Dynamic Compressive Response of Stainless-Steel Square Honeycombs, Journal of Applied Mechanics 74 (2007) 658-667.

[32] F. Cote, V. S. Deshpande, N. A. Fleck, A. G. Evans, The out-of-plane compressive behavior of metallic honeycombs, Materials Science and Engineering: A 380 (1-2) (2004) 272-280, ISSN 09215093.

[33] J. Brennan-Craddock, D. Brackett, R. Wildman, R. Hague, The design of impact absorbing structures for additive manufacture, Journal of Physics: Conference Series 382.

[34] G. Gray III, Classic Split-Hopkinson Pressure Bar Testing, ASM Handbook Vol 8: Mechanical Testing and Evaluation 8 (2000) 1041-1042.

[35] M. A. S. Metals, AISI Type 316L Stainless Steel, Annealed, URL http://asm.matweb.com/search/ SpecificMaterial.asp?bassnum=MQ316Q, 2014.

[36] C. Kamath, B. El-dasher, G. F. Gallegos, W. E. King, A. Sisto, Density of additively-manufactured, 316L SS parts using laser powder-bed fusion at powers up to $400 \mathrm{~W}$, The International Journal of Advanced Manufacturing Technology 74 (1-4) (2014) 65-78.

[37] E. Yasa, J. Kruth, Microstructural investigation of Selective Laser Melting 316L stainless steel parts exposed to laser re-melting, Procedia Engineering 19 (2011) 389-395.

[38] L. Thijs, F. Verhaeghe, T. Craeghs, J. V. Humbeeck, J.-P. Kruth, A study of the microstructural evolution during selective laser melting of Ti-6Al-4V, Acta Materialia 58 (9) (2010) 3303-3312.

[39] L. E. Murr, E. Martinez, K. N. Amato, S. M. Gaytan, J. Hernandez, D. A. Ramirez, P. W. Shindo, F. Medina, R. B. Wicker, Fabrication of Metal and Alloy Components by Additive Manufacturing: Examples of 3D Materials Science, Journal of Materials Research and Technology 1 (1) (2012) 42-54.

[40] E. Martinez, L. Murr, K. Amato, J. Hernandez, P. Shindo, S. Gaytan, D. Ramirez, F. Medina, R. Wicker, 3D Microstructural architectures for metal and alloy components fabricated by 3D printing/additive manufacturing technologies, 2012.

[41] T. Niendorf, S. Leuders, A. Riemer, H. A. Richard, T. Troster, D. Schwarze, Highly Anisotropic Steel Processed by Selective Laser Melting, Metallurgical and Materials Transactions B 44 (4) (2013) 794-796. 
[42] M. Rizal Alkahari, T. Furumoto, T. Ueda, A. Hosokawa, R. Tanaka, M. S. Abdul Aziz, Thermal conductivity of metal powder and consolidated material fabricated via selective laser melting, Key Engineering Materials 523-524 (2012) 244-249.

[43] B. Kashyap, K. Tangri, On the Hall-Petch relationship and substructural evolution in type 316L stainless steel, Acta Metallurgica 43 (11) (1995) 3971-3981.

[44] K. K. Singh, S. Sangal, G. S. Murty, Hall Petch behaviour of 316L austenitic stainless steel at room temperature, Materials Science and Technology 18 (2) (2002) 165-172.

[45] J. Hollomon, Properties and Structure of Steel - Tensile Deformation, AIME Transactions 162 (1945) 268-290.

[46] P. Ludwik, Elemente der technologischen mechanik, Julius Springer, Berlin, 1909.

[47] E. Voce, A practical strain hardening function, Metallurgia 51 (1955) 219.

[48] D. Ludwigson, Modified stress-strain relation for FCC metals and alloys, Metallurgical Transactions 2 (1971) $2825-2828$.

[49] R. Holloman, K. Kandan, V. Deshpande, H. Wadley, Dynamic compression of square tube cellular structures, Journal of Mechanics of Materials and Structures 9 (2) (2014) 149-182.

[50] J. Lifshitz;, H. Leber, Data processing in the split hopkinson pressure bar tests, International Journal of Impact Engineering 15 (6) (1994) 723-733.

[51] D. Bancroft, The velocity of longitudinal waves in cylindrical bars, Physical Review 59 (1941) 588-593.

[52] S. Reid, C. Peng, Dynamic Uniaxial Crushing of Wood, International Journal of Impact Engineering 19 (5-6) (1997) 531-570.

[53] H. Lindberg, A. Florence, Dynamic Pulse Buckling - Theory and Experiment, SRI International, 1983. 